OPEN ACCESS

Edited by:

Ayan Banerjee,

Indian Institute of Science Education and Research Kolkata, India

Reviewed by:

Francisco J. Sevilla,

Universidad Nacional Autónoma de

México, Mexico

Ignazio Licata,

Institute for Scientific Methodology

(ISEM), Italy

${ }^{*}$ Correspondence: Ralf Eichhorn

eichhorn@nordita.org

Specialty section:

This article was submitted to Interdisciplinary Physics,

a section of the journal

Frontiers in Physics

Received: 13 July 2020 Accepted: 07 October 2020

Published: 11 January 2021

Citation:

Dabelow L and Eichhorn R (2021) Irreversibility in Active Matter: General Framework for Active Ornstein-Uhlenbeck Particles.

Front. Phys. 8:582992.

doi: 10.3389/fphy.2020.582992

\section{Irreversibility in Active Matter: General Framework for Active Ornstein-Uhlenbeck Particles}

\author{
Lennart Dabelow ${ }^{1}$ and Ralf Eichhorn ${ }^{2 *}$ \\ ${ }^{1}$ Fakultät für Physik, Universität Bielefeld, Bielefeld, Germany, ${ }^{2}$ Nordita, Royal Institute of Technology and Stockholm University, \\ Stockholm, Sweden
}

Active matter systems are driven out of equilibrium by conversion of energy into directed motion locally on the level of the individual constituents. In the spirit of a minimal description, active matter is often modeled by so-called active Ornstein-Uhlenbeck particles an extension of passive Brownian motion where activity is represented by an additional fluctuating non-equilibrium "force" with simple statistical properties (OrnsteinUhlenbeck process). While in passive Brownian motion, entropy production along trajectories is well-known to relate to irreversibility in terms of the log-ratio of probabilities to observe a certain particle trajectory forward in time in comparison to observing its time-reversed twin trajectory, the connection between these concepts for active matter is less clear. It is therefore of central importance to provide explicit expressions for the irreversibility of active particle trajectories based on measurable quantities alone, such as the particle positions. In this technical note we derive a general expression for the irreversibility of AOUPs in terms of path probability ratios (forward vs. backward path), extending recent results from [PRX 9, 021009 (2019)] by allowing for arbitrary initial particle distributions and states of the active driving.

\section{Keywords: active matter, stochastic thermodynamics, non-equilibrium, active Brownian motion, active Ornstein- Uhlenbeck particle, irreversibility, path integrals}

\section{INTRODUCTION}

Irreversible thermodynamic processes are characterized by a positive entropy change in their "Universe", i.e., in the combined system of interest and its environment [1]. In macroscopic (equilibrium) thermodynamics, where entropy is a state variable, this change usually refers to the difference between the entropy in the final state of the "Universe" reached at the end of the process and in the initial state from where it started. In small mesoscopic systems on the micro- and nanometer scale, such as a colloidal Brownian particle diffusing in an aqueous solution, it has been established within the framework of stochastic thermodynamics [2-6] that the total entropy change should be evaluated from the entropy produced in the system and in its thermal environment along the specific trajectory the system follows during the process. This procedure remains valid even when the system is far from equilibrium, for example due to persistent currents or because it is driven by an external protocol realizing the thermodynamic process. The omnipresence of thermal fluctuations on the mesoscopic scale leads to a distribution of possible paths the system can take to go from the initial to the final state, and, accordingly to a distribution of entropy changes. A central result in stochastic thermodynamics is that the total entropy change $\Delta S$ along a specific realization of the system path 
(divided by Boltzmann's constant $k_{\mathrm{B}}$ ) equals the log-ratio of probabilities for observing that specific path vs. observing the same path in a time-reversed manner, i.e., traversing the same trajectory, but from the final state to the original initial state [2-5]. As a direct consequence, the total entropy change $\Delta S$ fulfills a so-called fluctuation theorem, $\left\langle\exp \left(-\Delta S / k_{\mathrm{B}}\right)\right\rangle=1$ (the angular brackets denote an average over all trajectories connecting the initial and final states), which can be viewed as a generalization of the second law of thermodynamics to the non-equilibrium realm when deviations from equilibrium are induced by externally applied forces or gradients.

A fundamentally different class of non-equilibrium systems are so-called "active particles", like Janus colloids with catalytic surfaces or bacteria [7-11], which have the ability to locally convert energy into self-propulsion, i.e., they move independently of external forces or thermal fluctuations. The source of non-equilibrium is the energy-to-motion conversion process on the level of the individual particle. This out-of-equilibrium process produces entropy, but the various degrees of freedom maintaining the self-propulsion are usually not observable in typical experiments with active particles, such that this entropy production can in general not be quantified. Moreover, for the (collective) behavior of active particles emerging from self-propulsion, as described, e.g., in Refs. $12-15$, the details of the propulsion mechanism and the amount of dissipation connected with it are largely irrelevant. In analogy to the stochastic thermodynamics of passive Brownian particles, a central question in active matter is therefore how the path probabilities for translational degrees of freedom of the active particles and the associated log-ratio of forward vs. backward path probabilities is connected to irreversibility and entropy production [16-18]. We remark that this is an ongoing debate $[16,19-24]$ which we will not resolve here. Rather, we will provide a central step toward an understanding of the role of the path probability ratio in active matter by providing exact analytical expressions for a simple but highly successful and well-established [15, 25-31] model of active matter, namely the active OrnsteinUhlenbeck particle (AOUP) [16, 18-21, 24, 32-44]. In this model, self-propulsion is realized via a fluctuating "driving force" in the equations of motion [7, 10] with Gaussian distribution and exponential time-correlation (see Section 2.1). By integrating out these active fluctuations, we derive an explicit analytical expression for the path weight of an AOUP, valid for arbitrary values of the model parameters, arbitrary finite duration of the particle trajectory and arbitrary initial distributions of particle positions and active fluctuations (see Section 4). Using this path weight, we then derive the irreversibility measure in form of the log-ratio of forward vs. backward path probabilities and comment on its physical implications (Section 5). Before establishing these general results, we briefly recall earlier findings from Ref. 18 for independent initial conditions of particle positions and active fluctuations, see Section 3. We conclude with a short discussion in Section 6, including potential applications of our results.

\section{SETUP}

\subsection{Model}

The model for an active Ornstein-Uhlenback particle (AOUP) consists in a standard overdamped Langevin equation for a passive Brownian particle at position $x$ in $d$ dimensions with an additional fluctuating force, which represents the active selfpropulsion and which we denote by $\sqrt{2 D_{\mathrm{a}}} \boldsymbol{\eta}(t)$,

$$
\dot{\boldsymbol{x}}(t)=\frac{1}{\gamma} \boldsymbol{f}(\boldsymbol{x}(t), t)+\sqrt{2 D_{a}} \boldsymbol{\eta}(t)+\sqrt{2 D} \boldsymbol{\xi}(t) .
$$

Here, the dot denotes the time-derivative, $\gamma$ is the viscous friction coefficient, $\boldsymbol{f}(\boldsymbol{x}, t)$ represents externally applied forces (conservative or non-conservative, and possibly timedependent). Furthermore, $\xi(t)$ are mutually independent Gaussian white noise sources modeling thermally fluctuating forces with $\delta$-correlation in time, i.e., $\left\langle\xi_{i}(t)\right\rangle=0$, $\left\langle\xi_{i}(t) \xi_{j}\left(t^{\prime}\right)\right\rangle=\delta_{i j} \delta\left(t-t^{\prime}\right)$, and $D$ is the particle diffusion coefficient, related to the temperature $T$ of the thermal bath via Einstein's relation $D=k_{\mathrm{B}} T / \gamma$. All bold-face letters represent $d$-dimensional vectors with components usually labeled by subscripts $i$, $j$, etc. In analogy to the thermal fluctuations, we denote the strength of the active fluctuations $\boldsymbol{\eta}(t)$ by $\sqrt{2 D_{\mathrm{a}}}$ with an active "diffusion coefficient" $D_{\mathrm{a}}$. For an AOUP, the active fluctuations follow a Gaussian process with exponential timecorrelations, which can be generated by a so-called OrnsteinUhlenbeck process,

$$
\dot{\boldsymbol{\eta}}(t)=-\frac{1}{\tau_{\mathrm{a}}} \boldsymbol{\eta}(t)+\frac{1}{\tau_{\mathrm{a}}} \boldsymbol{\zeta}(t),
$$

where $\tau_{\mathrm{a}}$ is the correlation time of the active noise fluctuations, i.e.,

$$
\left\langle\eta_{i}(t) \eta_{j}\left(t^{\prime}\right)\right\rangle=\frac{\delta_{i j}}{2 \tau_{\mathrm{a}}} e^{-\left|t-t^{\prime}\right| / \tau_{\mathrm{a}}} .
$$

\subsection{Central Quantity of Interest}

Our central goal is to evaluate the path weight $\mathfrak{p}\left[\underline{\boldsymbol{x}} \mid \boldsymbol{x}_{\mathrm{i}}\right]$ for particle positions alone, conditioned on the initial position $\boldsymbol{x}_{\mathrm{i}}$ for an arbitrary initial distribution $p_{\mathrm{i}}\left(\boldsymbol{\eta}_{\mathrm{i}} \mid \boldsymbol{x}_{\mathrm{i}}\right)$ of the active fluctuations given the specific value $\boldsymbol{x}_{\mathrm{i}}$. By definition, we can write this path weight as

$$
\mathfrak{p}\left[\underline{\boldsymbol{x}} \mid \boldsymbol{x}_{\mathrm{i}}\right]=\int \mathcal{D} \overline{\boldsymbol{\eta}} \mathfrak{p}\left[\underline{\boldsymbol{x}}, \underline{\boldsymbol{\eta}} \mid \boldsymbol{x}_{\mathrm{i}}, \boldsymbol{\eta}_{\mathrm{i}}\right] p_{\mathrm{i}}\left(\boldsymbol{\eta}_{\mathrm{i}} \mid \boldsymbol{x}_{\mathrm{i}}\right),
$$

where the path integral over $\overline{\boldsymbol{\eta}}:=\{\boldsymbol{\eta}(t)\}_{t=\tau_{\mathrm{i}}}^{\tau_{\mathrm{f}}}$ includes the initial configuration $\boldsymbol{\eta}_{\mathrm{i}}$, whereas the notation $\boldsymbol{\eta}:=\{\boldsymbol{\eta}(t)\}_{t>\tau_{\mathrm{i}}}^{\tau_{\mathrm{f}}}$ denotes the same history of active fluctuations without the initial configuration $\boldsymbol{\eta}_{\mathrm{i}}$, and similarly for $\underline{\boldsymbol{x}}:=\{\boldsymbol{x}(t)\}_{t>\tau_{\mathrm{i}}}^{\tau_{\mathrm{f}}}$. Moreover,

$$
\begin{aligned}
& \mathfrak{p}\left[\underline{\boldsymbol{x}}, \underline{\boldsymbol{\eta}} \mid \boldsymbol{x}_{\mathrm{i}}, \boldsymbol{\eta}_{\mathrm{i}}\right] \propto \exp \left\{-\int_{\tau_{\mathrm{i}}}^{\tau_{\mathrm{f}}} \mathrm{d} t\left[\frac{\left(\dot{\boldsymbol{x}}_{t}-\boldsymbol{v}_{t}-\sqrt{2 D_{\mathrm{a}}} \boldsymbol{\eta}_{t}\right)^{2}}{4 D}\right.\right. \\
& \left.\left.+\frac{\left(\tau_{\mathrm{a}} \dot{\boldsymbol{\eta}}_{t}+\boldsymbol{\eta}_{t}\right)^{2}}{2}+\frac{\nabla \cdot \boldsymbol{v}_{t}}{2}\right]\right\}
\end{aligned}
$$


is the standard Onsager-Machlup path weight [45-47] for the joint process $(\underline{\boldsymbol{x}}, \underline{\boldsymbol{\eta}})$, where we use the shorthand notation $\boldsymbol{v}_{t}=$ $\boldsymbol{f}_{t} / \gamma=\boldsymbol{f}(\boldsymbol{x}(t), t) / \gamma$ and $\boldsymbol{x}_{t} \equiv \boldsymbol{x}(t), \boldsymbol{\eta}_{t} \equiv \boldsymbol{\eta}(t)$, etc. The technical challenge consists in performing the integral over the active fluctuations $\bar{\eta}$ without explicitly specifying the initial distribution $p_{\mathrm{i}}\left(\boldsymbol{\eta}_{\mathrm{i}} \mid \boldsymbol{x}_{\mathrm{i}}\right)$.

\section{PATH WEIGHT FOR INDEPENDENT INITIAL CONDITIONS}

\subsection{The Results From Ref. 18}

We start by summarizing the main results from Ref. 18. In Ref. 18 we gave the path weight for trajectories $\overline{\boldsymbol{x}}=\{\boldsymbol{x}(t)\}_{t=0}^{\tau}$, running from initial time $\tau_{\mathrm{i}}=0$ to final time $\tau_{\mathrm{f}}=\tau$, assuming that the active noise is initially independent of the particle positions and in its steady state, i.e., $p_{\mathrm{i}}\left(\boldsymbol{\eta}_{0} \mid \boldsymbol{x}_{0}\right)=p_{\mathrm{ss}}\left(\boldsymbol{\eta}_{0}\right)=\sqrt{\tau_{\mathrm{a}} / \pi} \mathrm{e}^{-\tau_{\mathrm{a}} \boldsymbol{\eta}_{0}^{2}}$. We found

$$
\begin{aligned}
\mathfrak{p}_{(0, \tau]}^{\text {ind }}\left[\underline{\boldsymbol{x}} \mid \boldsymbol{x}_{0}\right] & \propto \exp \left\{-\frac{1}{4 D} \int_{0}^{\tau} \mathrm{d} t \int_{0}^{\tau} \mathrm{d} t^{\prime}\left[\dot{\boldsymbol{x}}_{t}-\boldsymbol{v}_{t}\right]^{T}\left[\delta\left(t-t^{\prime}\right)\right.\right. \\
\left.\left.-\frac{D_{\mathrm{a}}}{D} \Gamma_{[0, \tau]}^{\text {ind }}\left(t, t^{\prime}\right)\right]\left[\dot{\boldsymbol{x}}_{t^{\prime}}-\boldsymbol{v}_{t^{\prime}}\right]-\frac{1}{2} \int_{0}^{\tau} \mathrm{d} t \nabla \cdot \boldsymbol{v}_{t}\right\} &
\end{aligned}
$$

with the memory kernel

$$
\begin{aligned}
& \Gamma_{[0, \tau]}^{\text {ind }}\left(t, t^{\prime}\right):=\left(\frac{1}{2 \tau_{\mathrm{a}}^{2} \lambda}\right) \\
& \times \frac{\kappa_{+}^{2} \mathrm{e}^{-\lambda\left|t-t^{\prime}\right|}+\kappa_{-}^{2} \mathrm{e}^{-\lambda\left(2 \tau-\left|t-t^{\prime}\right|\right)}-\kappa_{+} \kappa_{-}\left[\mathrm{e}^{-\lambda\left(t+t^{\prime}\right)}+\mathrm{e}^{-\lambda\left(2 \tau-t-t^{\prime}\right)}\right]}{\kappa_{+}^{2}-\kappa_{-}^{2} \mathrm{e}^{-2 \lambda \tau}},
\end{aligned}
$$

where $\lambda:=\sqrt{1+D_{\mathrm{a}} / D} / \tau_{\mathrm{a}}$ and $\kappa_{ \pm}:=1 \pm \sqrt{1+D_{\mathrm{a}} / D}$.

\subsection{Stationary-State Scenario}

If we have a trajectory $\overline{\boldsymbol{x}}=\{\boldsymbol{x}(t)\}_{t=\tau_{\mathrm{i}}}^{\tau_{\mathrm{f}}}$ running from arbitrary times $\tau_{\mathrm{i}}$ to $\tau_{\mathrm{f}}$ instead, we can shift time as $t \rightarrow t-\tau_{\mathrm{i}}$ and identify $\tau=\tau_{\mathrm{f}}-$ $\tau_{\mathrm{i}}$ as the duration of the trajectory to convert $(0, \tau]$ path weights to those running from $\tau_{\mathrm{i}}$ to $\tau_{\mathrm{f}}$. Performing these replacements, the memory kernel Eq. 7 turns into

$$
\begin{aligned}
& \Gamma_{\left[\tau_{\mathrm{i}}, \tau_{\mathrm{f}}\right]}^{\mathrm{ind}}\left(t, t^{\prime}\right):=\left(\frac{1}{2 \tau_{\mathrm{a}}^{2} \lambda}\right) \\
& \times \frac{\kappa_{+}^{2} \mathrm{e}^{-\lambda\left|t-t^{\prime}\right|}+\kappa_{-}^{2} \mathrm{e}^{-\lambda\left[2\left(\tau_{\mathrm{f}}-\tau_{\mathrm{i}}\right)-\left|t-t^{\prime}\right|\right]}-\kappa_{+} \kappa_{-}\left[\mathrm{e}^{-\lambda\left(t+t^{\prime}-2 \tau_{\mathrm{i}}\right)}+\mathrm{e}^{-\lambda\left(2 \tau_{\mathrm{f}}-t-t^{\prime}\right)}\right]}{\kappa_{+}^{2}-\kappa_{-}^{2} \mathrm{e}^{-2 \lambda\left(\tau_{\mathrm{f}}-\tau_{\mathrm{i}}\right)}} .
\end{aligned}
$$

Consequently, the corresponding path weight for a trajectory starting at $x_{\mathrm{i}}$ at time $\tau_{\mathrm{i}}$ reads

$$
\begin{aligned}
& \mathfrak{p}_{\left(\tau_{\mathrm{i}}, \tau_{\mathrm{f}}\right.}^{\text {ind }}\left[\underline{\boldsymbol{x}} \mid \boldsymbol{x}_{\mathrm{i}}\right] \propto \exp \left\{-\frac{1}{4 D} \int_{\tau_{\mathrm{i}}}^{\tau_{\mathrm{f}}} \mathrm{d} t \int_{\tau_{\mathrm{i}}}^{\tau_{\mathrm{f}}} \mathrm{d} t^{\prime}\left[\dot{\boldsymbol{x}}_{t}-\boldsymbol{v}_{t}\right]^{T}\left[\delta\left(t-t^{\prime}\right)\right.\right. \\
&\left.\left.-\frac{D_{\mathrm{a}}}{D} \Gamma_{\left[\tau_{\mathrm{i}}, \tau_{\mathrm{f}}\right]}^{\text {ind }}\left(t, t^{\prime}\right)\right]\left[\dot{\boldsymbol{x}}_{t^{\prime}}-\boldsymbol{v}_{t^{\prime}}\right]-\frac{1}{2} \int_{\tau_{\mathrm{i}}}^{\tau_{\mathrm{f}}} \mathrm{d} t \nabla \cdot \boldsymbol{v}_{t}\right\}
\end{aligned}
$$

Letting $\tau_{\mathrm{i}} \rightarrow-\infty$ (stationary-state scenario), the memory kernel becomes

$$
\Gamma_{\left(-\infty, \tau_{\mathrm{f}}\right]}^{\mathrm{ind}}\left(t, t^{\prime}\right)=\frac{1}{2 \tau_{\mathrm{a}}^{2} \lambda}\left[\mathrm{e}^{-\lambda\left|t-t^{\prime}\right|}-\frac{\kappa_{-}}{\kappa_{+}} \mathrm{e}^{-\lambda\left(2 \tau_{\mathrm{f}}-t-t^{\prime}\right)}\right] .
$$

For "infinitely long" stationary-state trajectories, for which also $\tau_{\mathrm{f}} \rightarrow \infty$, this expression further reduces to

$$
\Gamma_{(-\infty, \infty)}^{\text {ind }}\left(t, t^{\prime}\right)=\frac{1}{2 \tau_{\mathrm{a}}^{2} \lambda} \mathrm{e}^{-\lambda\left|t-t^{\prime}\right|} .
$$

The latter special case has been derived independently in Ref. 24 via Fourier transformation, see eq. 25 in Ref. 24, in order to analyze "entropy production" based on path-probability ratios. Similar Fourier-transform techniques for Langevin systems have been used in Ref. 48 for deriving a fluctuation relation at large times, with findings for the non-local "inverse temperature" as integration kernel in the "entropy production" corresponding to those in Ref. 24, and to our Eq. 11.

\section{PATH WEIGHT FOR ARBITRARY INITIAL CONDITIONS}

In this section, we generalize the path weight Eq. 9 to allow for arbitrary joint initial distributions $p_{\mathrm{i}}\left(\boldsymbol{x}_{\mathrm{i}}, \boldsymbol{\eta}_{\mathrm{i}}\right)$ of particle positions and active fluctuations. Keeping in mind that we can time-shift final results between trajectories running during a time interval $(0, \tau]$ and during arbitrary intervals $\left(\tau_{\mathrm{i}}, \tau_{\mathrm{f}}\right]$ as in Section 3.2, we here consider without loss of generality trajectories with $\tau_{\mathrm{i}}=0$ and $\tau_{\mathrm{f}}=\tau$. For notational simplicity we drop the subscripts $(0, \tau]$ or $[0, \tau]$ on $\mathfrak{p}$ and $\Gamma$.

We start in Section 4.1 by first calculating $\Gamma$ for a general Gaussian initial distribution of $\boldsymbol{\eta}_{0}$ independent of $\boldsymbol{x}_{0}$, which has variance $\sigma^{2}$ and is centered at $\widehat{\boldsymbol{\eta}}_{0}$,

$$
p_{\widehat{\boldsymbol{\eta}}_{0}, \sigma}\left(\boldsymbol{\eta}_{0}\right)=\frac{1}{\sqrt{2 \pi \sigma^{2}}} \mathrm{e}^{-\left(\boldsymbol{\eta}_{0}-\widehat{\boldsymbol{\eta}}_{0}\right)^{2}} / 2 \sigma^{2} .
$$

Then, in Section 4.2, we show how this result can be used to cover any arbitrary initial distribution $p_{\mathrm{i}}\left(\boldsymbol{\eta}_{0} \mid \boldsymbol{x}_{0}\right)$.

\subsection{Gaussian Initial Distribution}

With Eqs 4 and 5, and the initial distribution $p_{\mathrm{i}}\left(\boldsymbol{\eta}_{\mathrm{i}} \mid \boldsymbol{x}_{\mathrm{i}}\right)=$ $p_{\mathrm{i}}\left(\boldsymbol{\eta}_{0} \mid \boldsymbol{x}_{0}\right)=p_{\mathrm{i}}\left(\boldsymbol{\eta}_{0}\right)=p_{\widehat{\boldsymbol{\eta}}_{0}, \sigma}\left(\boldsymbol{\eta}_{0}\right)$ from Eq. 12, the path weight we want to evaluate reads

$$
\begin{aligned}
\mathfrak{p}_{\widehat{\boldsymbol{\eta}}_{0}, \sigma}^{\text {ind }}\left[\underline{\boldsymbol{x}} \mid \boldsymbol{x}_{0}\right] \propto \frac{1}{\sqrt{2 \pi \sigma^{2}}} \int \mathcal{D} \overline{\boldsymbol{\eta}} \\
\times \exp \left\{-\int_{0}^{\tau} \mathrm{d} t\left[\frac{\left(\dot{\boldsymbol{x}}_{t}-\boldsymbol{v}_{t}-\sqrt{2 D_{\mathrm{a}}} \boldsymbol{\eta}_{t}\right)^{2}}{4 D}+\frac{\left(\tau_{\mathrm{a}} \dot{\boldsymbol{\eta}}_{t}+\boldsymbol{\eta}_{t}\right)^{2}}{2}+\frac{\nabla \cdot \boldsymbol{v}_{t}}{2}\right]\right. \\
\left.\quad-\frac{\left(\boldsymbol{\eta}_{0}-\widehat{\boldsymbol{\eta}}_{0}\right)^{2}}{2 \sigma^{2}}\right\}
\end{aligned}
$$

The superscript "ind" emphasizes again that we use statistically independent initial conditions for $\boldsymbol{x}_{0}$ and $\boldsymbol{\eta}_{0}$. After partial integration of the $\dot{\boldsymbol{\eta}}_{t}$ terms, similarly as in Ref. 18, we can express the path integral as 


$$
\begin{aligned}
& \mathfrak{p}_{\widehat{\boldsymbol{\eta}}_{0}, \sigma}^{\text {ind }}\left[\underline{\boldsymbol{x}} \mid \boldsymbol{x}_{0}\right] \propto \frac{1}{\sqrt{2 \pi \sigma^{2}}} \exp \left\{-\int_{0}^{\tau} \mathrm{d} t\left[\frac{\left(\dot{\boldsymbol{x}}_{t}-\boldsymbol{v}_{t}\right)^{2}}{4 D}+\frac{\nabla \cdot \boldsymbol{v}_{t}}{2}\right]-\frac{\widehat{\boldsymbol{\eta}}_{0}^{2}}{2 \sigma^{2}}\right\} \\
& \quad \times \int \mathcal{D} \overline{\boldsymbol{\eta}} \exp \left\{-\frac{1}{2} \int_{0}^{\tau} \mathrm{d} t \int_{0}^{\tau} \mathrm{d} t^{\prime} \boldsymbol{\eta}_{t}^{T} V_{\sigma}\left(t, t^{\prime}\right) \boldsymbol{\eta}_{t^{\prime}}\right. \\
& \left.+\int_{0}^{\tau} \mathrm{d} t \boldsymbol{\eta}_{t}^{T}\left[\frac{\sqrt{2 D_{\mathrm{a}}}}{2 D}\left(\dot{\boldsymbol{x}}_{t}-\boldsymbol{v}_{t}\right)+\delta(t) \frac{\widehat{\boldsymbol{\eta}}_{0}}{\sigma^{2}}\right]\right\}
\end{aligned}
$$

with the differential operator

$$
\begin{aligned}
& V_{\sigma}\left(t, t^{\prime}\right):=\delta\left(t-t^{\prime}\right)\left[-\tau_{\mathrm{a}}^{2} \partial_{t^{\prime}}^{2}+1+\frac{D_{\mathrm{a}}}{D}+\delta\left(t^{\prime}\right)\left(-\tau_{\mathrm{a}}^{2} \partial_{t^{\prime}}-\tau_{\mathrm{a}}+\frac{1}{\sigma^{2}}\right)\right. \\
& \left.+\delta\left(\tau-t^{\prime}\right)\left(\tau_{\mathrm{a}}^{2} \partial_{t^{\prime}}+\tau_{\mathrm{a}}\right)\right] .
\end{aligned}
$$

Performing the Gaussian integral over $\overline{\boldsymbol{\eta}}$ in Eq. 14, we obtain

$$
\begin{aligned}
& \mathfrak{p}_{\widehat{\boldsymbol{\eta}}_{0}, \sigma}^{\text {ind }}[\left.\underline{\boldsymbol{x}} \mid \boldsymbol{x}_{0}\right] \propto \frac{\left(\operatorname{Det} V_{\sigma}\right)^{-1 / 2}}{\sqrt{2 \pi \sigma^{2}}} \exp \left\{-\frac{1}{4 D} \int_{0}^{\tau} \mathrm{d} t \int_{0}^{\tau} \mathrm{d} t^{\prime}\left(\dot{\boldsymbol{x}}_{t}-\boldsymbol{v}_{t}\right)^{\mathrm{T}}\right. \\
& \times {\left[\delta\left(t-t^{\prime}\right)-\frac{D_{\mathrm{a}}}{D} \Gamma_{\sigma}\left(t, t^{\prime}\right)\right]\left(\dot{\boldsymbol{x}}_{t^{\prime}}-\boldsymbol{v}_{t^{\prime}}\right) } \\
&+\int_{0}^{\tau} \mathrm{d} t\left[\frac{\sqrt{2 D_{\mathrm{a}}}}{2 D}\left(\dot{\boldsymbol{x}}_{t}-\boldsymbol{v}_{t}\right)^{T} \frac{\Gamma_{\sigma}(t, 0)}{\sigma^{2}} \widehat{\boldsymbol{\eta}}_{0}-\frac{\nabla \cdot \boldsymbol{v}_{t}}{2}\right] \\
&\left.+\left[\frac{\Gamma_{\sigma}(0,0)}{\sigma^{2}}-1\right] \frac{\widehat{\boldsymbol{\eta}}_{0}^{2}}{2 \sigma^{2}}\right\},
\end{aligned}
$$

where $\Gamma_{\sigma}\left(t, t^{\prime}\right)$ denotes the operator inverse of $V_{\sigma}\left(t, t^{\prime}\right)$ in the sense that $\int_{0}^{\tau} \mathrm{d} t^{\prime} V_{\sigma}\left(t, t^{\prime}\right) \Gamma_{\sigma}\left(t^{\prime}, t^{\prime \prime}\right)=\delta\left(t-t^{\prime \prime}\right)$. It can be constructed similarly to the procedure in Ref. 18. In particular, we can also write $\Gamma_{\sigma}\left(t, t^{\prime}\right)=G\left(t, t^{\prime}\right)+H_{\sigma}\left(t, t^{\prime}\right)$. Here $G\left(t, t^{\prime}\right)$ is the Green's function defined by $\left[-\tau_{a}^{2} \partial_{t}^{2}+\left(1+D_{\mathrm{a}} / D\right)\right] G\left(t, t^{\prime}\right)=$ $\delta\left(t-t^{\prime}\right)$ and $G\left(0, t^{\prime}\right)=G\left(\tau, t^{\prime}\right)=0$. The second ingredient, $H\left(t, t^{\prime}\right)$, is a solution of the associated homogeneous problem, $\left[-\tau_{a}^{2} \partial_{t}^{2}+\left(1+D_{\mathrm{a}} / D\right)\right] H\left(t, t^{\prime}\right)=0$, fixing the boundary terms as prescribed by Eq. 15. More details are given the Appendix. We find

$$
\begin{aligned}
& \Gamma_{\sigma}\left(t, t^{\prime}\right)=\left(\frac{1}{2 \tau_{\mathrm{a}}^{2} \lambda}\right)\left[\kappa_{+}\left(1-\sigma^{2} \tau_{\mathrm{a}} \kappa_{-}\right)-\kappa_{-}\left(1-\sigma^{2} \tau_{\mathrm{a}} \kappa_{+}\right) \mathrm{e}^{-2 \lambda \tau}\right]^{-1} \\
& \times\left[\kappa_{+}\left(1-\sigma^{2} \tau_{\mathrm{a}} \kappa_{-}\right) \mathrm{e}^{-\lambda\left|t-t^{\prime}\right|}+\kappa_{-}\left(1-\sigma^{2} \tau_{\mathrm{a}} \kappa_{+}\right) \mathrm{e}^{-\lambda\left(2 \tau-\left|t-t^{\prime}\right|\right.}\right) \\
& -\kappa_{+}\left(1-\sigma^{2} \tau_{\mathrm{a}} \kappa_{+}\right) \mathrm{e}^{-\lambda\left(t+t^{\prime}\right)}-\kappa_{-}\left(1-\sigma^{2} \tau_{\mathrm{a}} \kappa_{-}\right) \mathrm{e}^{\left.-\lambda\left(2 \tau-t-t^{\prime}\right)\right]}
\end{aligned}
$$

We note that Eq. 12 includes the steady-state distribution, $p_{\mathrm{ss}}\left(\boldsymbol{\eta}_{0}\right)=\sqrt{\tau_{\mathrm{a}} / \pi} \mathrm{e}^{-\tau_{\mathrm{a}}} \eta_{0}^{2}$, which arises for the active noise when evolving independently of the Brownian particle, as a special case for $\widehat{\boldsymbol{\eta}}_{0}=0$ and $\sigma^{2}=1 /\left(2 \tau_{\mathrm{a}}\right)$. Accordingly, we recover Eqs 6 and 7 when plugging $\widehat{\boldsymbol{\eta}}_{0}=0$ and $\sigma^{2}=1 /\left(2 \tau_{\mathrm{a}}\right)$ into Eqs 16 and 17, using $1-\kappa_{ \pm} / 2=\kappa_{\mp} / 2$.

\subsection{Arbitrary Initial Distribution}

To cover arbitrary initial distributions $p_{\mathrm{i}}\left(\boldsymbol{\eta}_{\mathrm{i}} \mid \boldsymbol{x}_{\mathrm{i}}\right)=p_{\mathrm{i}}\left(\boldsymbol{\eta}_{0} \mid \boldsymbol{x}_{0}\right)$ in $\boldsymbol{\eta}_{0}$, we introduce a $\delta$-distribution of the form $\delta\left(\boldsymbol{\eta}_{0}-\right.$ $\left.\widehat{\boldsymbol{\eta}}_{0}\right)=\lim _{\sigma \rightarrow 0} \mathrm{e}^{-\left(\boldsymbol{\eta}_{0}-\widehat{\boldsymbol{\eta}}_{0}\right)^{2} / 2 \sigma^{2}} / \sqrt{2 \pi \sigma^{2}}$ and rewrite Eq. 4 (with $\tau_{\mathrm{i}}=0$, $\left.\tau_{\mathrm{f}}=\tau\right)$ as

$$
\begin{aligned}
& \mathfrak{p}\left[\underline{\boldsymbol{x}} \mid \boldsymbol{x}_{0}\right]=\int \mathcal{D} \overline{\boldsymbol{\eta}} \mathfrak{p}\left[\underline{\boldsymbol{x}}, \underline{\boldsymbol{\eta}} \mid \boldsymbol{x}_{0}, \boldsymbol{\eta}_{0}\right] p_{\mathrm{i}}\left(\boldsymbol{\eta}_{0} \mid \boldsymbol{x}_{0}\right) \\
& =\int \mathcal{D} \overline{\boldsymbol{\eta}} \mathfrak{p}\left[\underline{\boldsymbol{x}}, \underline{\boldsymbol{\eta}} \mid \boldsymbol{x}_{0}, \boldsymbol{\eta}_{0}\right] \int \mathrm{d} \widehat{\boldsymbol{\eta}}_{0} \delta\left(\boldsymbol{\eta}_{0}-\widehat{\boldsymbol{\eta}}_{0}\right) p_{\mathrm{i}}\left(\widehat{\boldsymbol{\eta}}_{0} \mid \boldsymbol{x}_{0}\right) \\
& =\lim _{\sigma \rightarrow 0} \int \mathrm{d} \widehat{\boldsymbol{\eta}}_{0} p_{\mathrm{i}}\left(\widehat{\boldsymbol{\eta}}_{0} \mid \boldsymbol{x}_{0}\right)\left[\frac{1}{\sqrt{2 \pi \sigma^{2}}} \int \mathcal{D} \overline{\boldsymbol{\eta}} \mathfrak{p}\left[\underline{\boldsymbol{x}}, \underline{\boldsymbol{\eta}} \mid \boldsymbol{x}_{0}, \boldsymbol{\eta}_{0}\right] \mathrm{e}^{-\left(\boldsymbol{\eta}_{0}-\widehat{\boldsymbol{\eta}}_{0}\right)^{2} / 2 \sigma^{2}}\right] .
\end{aligned}
$$

In view of Eq. 5 we see that the term in brackets is exactly $\mathfrak{p}_{\widehat{\boldsymbol{\eta}}_{0}, \sigma}^{\text {ind }}\left[\underline{\boldsymbol{x}} \mid \boldsymbol{x}_{0}\right]$ as defined in Eq. 13. Since we can also write $\mathfrak{p}\left[\underline{\boldsymbol{x}} \mid \boldsymbol{x}_{0}\right]=\int \mathrm{d} \widehat{\boldsymbol{\eta}}_{0} \mathfrak{p}\left[\underline{\boldsymbol{x}}, \widehat{\boldsymbol{\eta}}_{0} \mid \boldsymbol{x}_{0}\right]=\int \mathrm{d} \widehat{\boldsymbol{\eta}}_{0} p_{\mathrm{i}}\left(\widehat{\boldsymbol{\eta}}_{0} \mid \boldsymbol{x}_{0}\right) \mathfrak{p}\left[\underline{\boldsymbol{x}} \mid \boldsymbol{x}_{0}, \widehat{\boldsymbol{\eta}}_{0}\right] \quad$ we conclude that

$$
\mathfrak{p}\left[\underline{\boldsymbol{x}} \mid \boldsymbol{x}_{0}, \widehat{\boldsymbol{\eta}}_{0}\right]=\lim _{\sigma \rightarrow 0} \mathfrak{p}_{\widehat{\boldsymbol{\eta}}_{0}, \sigma}^{\mathrm{ind}}\left[\underline{\boldsymbol{x}} \mid \boldsymbol{x}_{0}\right]
$$

is the path weight conditioned on an initial position $\boldsymbol{x}_{0}$ and initial state of the active noise $\widehat{\boldsymbol{\eta}}_{0}$ with arbitrary distributions.

With the explicit result Eq. 16 for $\mathfrak{p}_{\widehat{\boldsymbol{\eta}}_{0, \sigma}}^{\text {ind }}\left[\underline{\boldsymbol{x}} \mid \boldsymbol{x}_{0}\right]$ we thus see that we have to calculate the $\sigma \rightarrow 0$ limit of the expressions $\left(\sigma^{2} \operatorname{Det} V_{\sigma}\right)^{1 / 2},\left[\Gamma_{\sigma}(t, 0) / \sigma^{2}-1\right] / \sigma^{2}, \Gamma_{\sigma}(0,0) / \sigma^{2}$ and $\Gamma_{\sigma}\left(t, t^{\prime}\right)$. From Eq. 15 we observe that $\sigma^{2} V_{\sigma}$ has a constant term (independent of $\sigma)$ and contributions quadratic in $\sigma$ such that $\left(\sigma^{2} \operatorname{Det} V_{\sigma}\right)^{1 / 2}$ reduces to an (irrelevant) constant as $\sigma \rightarrow 0$. Next, setting $t^{\prime}=0$ in Eq. 17 and using $\tau_{\mathrm{a}} \kappa_{+}-\tau_{\mathrm{a}} \kappa_{-}=2 \tau_{\mathrm{a}}^{2} \lambda$ we get

$$
\begin{aligned}
\frac{\Gamma_{\sigma}(t, 0)}{\sigma^{2}} & =\frac{\kappa_{+} \mathrm{e}^{-\lambda t}-\kappa_{-} \mathrm{e}^{-\lambda(2 \tau-t)}}{\kappa_{+}\left(1-\sigma^{2} \tau_{\mathrm{a}} \kappa_{-}\right)-\kappa_{-}\left(1-\sigma^{2} \tau_{\mathrm{a}} \kappa_{+}\right) \mathrm{e}^{-2 \lambda \tau}} \\
& \stackrel{\sigma \rightarrow 0}{\rightarrow} \frac{\kappa_{+} \mathrm{e}^{-\lambda t}-\kappa_{-} \mathrm{e}^{-\lambda(2 \tau-t)}}{\kappa_{+}-\kappa_{-} \mathrm{e}^{-2 \lambda \tau}} .
\end{aligned}
$$

If $t=0$, too, we obtain

$$
\frac{\Gamma_{\sigma}(0,0)}{\sigma^{2}}=\frac{\kappa_{+}-\kappa_{-} \mathrm{e}^{-2 \lambda \tau}}{\kappa_{+}\left(1-\sigma^{2} \tau_{\mathrm{a}} \kappa_{-}\right)-\kappa_{-}\left(1-\sigma^{2} \tau_{\mathrm{a}} \kappa_{+}\right) \mathrm{e}^{-2 \lambda \tau}},
$$

such that

$$
\begin{aligned}
{\left[\frac{\Gamma_{\sigma}(0,0)}{\sigma^{2}}-1\right] \frac{1}{\sigma^{2}} } & =\frac{\kappa_{+} \kappa_{-} \tau_{\mathrm{a}}\left(1-\mathrm{e}^{-2 \lambda \tau}\right)}{\kappa_{+}\left(1-\sigma^{2} \tau_{\mathrm{a}} \kappa_{-}\right)-\kappa_{-}\left(1-\sigma^{2} \tau_{\mathrm{a}} \kappa_{+}\right) \mathrm{e}^{-2 \lambda \tau}} \\
& \stackrel{\sigma \rightarrow 0}{\rightarrow} \frac{\kappa_{+} \kappa_{-} \tau_{\mathrm{a}}\left(1-\mathrm{e}^{-2 \lambda \tau}\right)}{\kappa_{+}-\kappa_{-} \mathrm{e}^{-2 \lambda \tau}}
\end{aligned}
$$

Furthermore, we define

$$
\begin{gathered}
\Gamma\left(t, t^{\prime}\right):=\lim _{\sigma \rightarrow 0} \Gamma_{\sigma}\left(t, t^{\prime}\right)=\left(\frac{1}{2 \tau_{\mathrm{a}}^{2} \lambda}\right) \\
\times \frac{\kappa_{+} \mathrm{e}^{-\lambda\left|t-t^{\prime}\right|}+\kappa_{-} \mathrm{e}^{-\lambda\left(2 \tau-\left|t-t^{\prime}\right|\right)}-\kappa_{+} \mathrm{e}^{-\lambda\left(t+t^{\prime}\right)}-\kappa_{-} \mathrm{e}^{-\lambda\left(2 \tau-t-t^{\prime}\right)}}{\kappa_{+}-\kappa_{-} \mathrm{e}^{-2 \lambda \tau}}
\end{gathered}
$$

as the memory kernel for the path weight conditioned on an arbitrary initial configuration $\left(\boldsymbol{x}_{0}, \widehat{\boldsymbol{\eta}}_{0}\right)$ of particle positions and active fluctuations. Altogether, Eq. 19 for this path weight then becomes 


$$
\begin{gathered}
\mathfrak{p}\left[\underline{\boldsymbol{x}} \mid \boldsymbol{x}_{0}, \widehat{\boldsymbol{\eta}}_{0}\right] \propto \exp \left\{-\frac{1}{4 D} \int_{0}^{\tau} \mathrm{d} t \int_{0}^{\tau} \mathrm{d} t^{\prime}\left(\dot{\boldsymbol{x}}_{t}-\boldsymbol{v}_{t}\right)^{T}\left[\delta\left(t-t^{\prime}\right)\right.\right. \\
\left.-\frac{D_{\mathrm{a}}}{D} \Gamma\left(t, t^{\prime}\right)\right]\left(\dot{\boldsymbol{x}}_{t^{\prime}}-\boldsymbol{v}_{t^{\prime}}\right) \\
+\int_{0}^{\tau} \mathrm{d} t\left[\frac{\sqrt{2 D_{\mathrm{a}}}}{2 D}\left(\dot{\boldsymbol{x}}_{t}-\boldsymbol{v}_{t}\right)^{T} \frac{\kappa_{+} \mathrm{e}^{-\lambda t}-\kappa_{-} \mathrm{e}^{-\lambda(2 \tau-t)}}{\kappa_{+}-\kappa_{-} \mathrm{e}^{-2 \lambda \tau}} \widehat{\boldsymbol{\eta}}_{0}-\frac{\nabla \cdot \boldsymbol{v}_{t}}{2}\right] \\
-\frac{D_{\mathrm{a}}}{2 D}\left[\frac{\tau_{\mathrm{a}}\left(1-\mathrm{e}^{-2 \lambda \tau}\right)}{\left.\kappa_{+}-\kappa_{-} \mathrm{e}^{-2 \lambda \tau}\right]} \widehat{\boldsymbol{\eta}}_{0}^{2}\right\},
\end{gathered}
$$

where we have used $\kappa_{+} \kappa_{-}=-D_{\mathrm{a}} / D$ in the fourth line.

Finally, we can shift trajectories similarly as in Section 3.2 to obtain the path weight for arbitrary trajectories $\overline{\boldsymbol{x}}=\{\boldsymbol{x}(t)\}_{t=\tau_{\mathrm{i}}}^{\tau_{\mathrm{f}}}$ conditioned on the joint initial state $\left(\boldsymbol{x}_{\mathrm{i}}, \boldsymbol{\eta}_{\mathrm{i}}\right)$ of position and active noise,

$$
\begin{gathered}
\mathfrak{p}_{\left(\tau_{\mathrm{i}}, \tau_{\mathrm{f}}\right]}\left[\underline{\boldsymbol{x}} \mid \boldsymbol{x}_{\mathrm{i}}, \boldsymbol{\eta}_{\mathrm{i}}\right] \propto \exp \left\{-\frac{1}{4 D} \int_{\tau_{\mathrm{i}}}^{\tau_{\mathrm{f}}} \mathrm{d} t \int_{\tau_{\mathrm{i}}}^{\tau_{\mathrm{f}}} \mathrm{d} t^{\prime}\left[\dot{\boldsymbol{x}}_{t}-\boldsymbol{v}_{t}\right]^{T}\left[\delta\left(t-t^{\prime}\right)\right.\right. \\
\left.-\frac{D_{\mathrm{a}}}{D} \Gamma_{\left[\tau_{\mathrm{i}}, \tau_{\mathrm{f}}\right]}\left(t, t^{\prime}\right)\right]\left[\dot{\boldsymbol{x}}_{t^{\prime}}-\boldsymbol{v}_{t^{\prime}}\right] \\
+\int_{\tau_{\mathrm{i}}}^{\tau_{\mathrm{f}}} \mathrm{d} t\left[\frac{\sqrt{2 D_{\mathrm{a}}}}{2 D}\left[\dot{\boldsymbol{x}}_{t}-\boldsymbol{v}_{t}\right] \frac{\mathrm{T} \kappa_{+} \mathrm{e}^{-\lambda\left(t-\tau_{\mathrm{i}}\right)}-\kappa_{-} \mathrm{e}^{-\lambda\left(2 \tau_{\mathrm{f}}-t-\tau_{\mathrm{i}}\right)}}{\left.\kappa_{+}-\kappa_{-} \mathrm{e}^{-2 \lambda\left(\tau_{\mathrm{f}}-\tau_{\mathrm{i}}\right)} \boldsymbol{\eta}_{\mathrm{i}}-\frac{\nabla \cdot \boldsymbol{v}_{t}}{2}\right]}\right. \\
\left.-\frac{D_{\mathrm{a}}}{2 D}\left[\frac{\tau_{\mathrm{a}}\left(1-\mathrm{e}^{-2 \lambda\left(\tau_{\mathrm{f}}-\tau_{\mathrm{i}}\right)}\right)}{\left.\kappa_{+}-\kappa_{-} \mathrm{e}^{-2 \lambda\left(\tau_{\mathrm{f}}-\tau_{\mathrm{i}}\right)}\right]}\right] \boldsymbol{\eta}_{\mathrm{i}}^{2}\right\}
\end{gathered}
$$

with

$$
\begin{aligned}
& \Gamma_{\left[\tau_{\mathrm{i}}, \tau_{\mathrm{f}}\right]}\left(t, t^{\prime}\right):=\left(\frac{1}{2 \tau_{\mathrm{a}}^{2} \lambda}\right) \\
& \times \frac{\kappa_{+} \mathrm{e}^{-\lambda\left|t-t^{\prime}\right|}+\kappa_{-} \mathrm{e}^{-\lambda\left(2\left(\tau_{\mathrm{f}}-\tau_{\mathrm{i}}\right)-\left|t-t^{\prime}\right|\right)}-\kappa_{+} \mathrm{e}^{-\lambda\left(t+t^{\prime}-2 \tau_{\mathrm{i}}\right)}-\kappa_{-} \mathrm{e}^{-\lambda\left(2 \tau_{\mathrm{f}}-t-t^{\prime}\right)}}{\kappa_{+}-\kappa_{-} \mathrm{e}^{-2 \lambda\left(\tau_{\mathrm{f}}-\tau_{\mathrm{i}}\right)}} .
\end{aligned}
$$

Given an initial distribution $p_{\mathrm{i}}\left(\boldsymbol{\eta}_{\mathrm{i}} \mid \boldsymbol{x}_{\mathrm{i}}\right)$ of the active fluctuations conditioned on the initial particle position, we can then compute the position-only path weight of an arbitrary trajectory by averaging over $p_{\mathrm{i}}\left(\boldsymbol{\eta}_{\mathrm{i}} \mid \boldsymbol{x}_{\mathrm{i}}\right)$,

$$
\mathfrak{p}_{\left(\tau_{\mathrm{i}}, \tau_{\mathrm{f}}\right]}\left[\underline{\boldsymbol{x}} \mid \boldsymbol{x}_{\mathrm{i}}\right]=\int \mathrm{d} \boldsymbol{\eta}_{\mathrm{i}} \mathfrak{p}_{\left(\tau_{\mathrm{i}}, \tau_{\mathrm{f}}\right]}\left[\underline{\boldsymbol{x}} \mid \boldsymbol{x}_{\mathrm{i}}, \boldsymbol{\eta}_{\mathrm{i}}\right] p_{\mathrm{i}}\left(\boldsymbol{\eta}_{\mathrm{i}} \mid \boldsymbol{x}_{\mathrm{i}}\right) .
$$

Equations 25-27 represent the first central result of the present contribution, a general expression for the path weight of active Ornstein-Uhlenbeck particles in position space only, for arbitrary trajectories with arbitrary initial and final times and arbitrary initial distributions. There is no approximation involved, so that our results are valid for any values of thermal and active noise parameters $D$ and $D_{\mathrm{a}}, \tau_{\mathrm{a}}$.

We expect that the specific initial configuration becomes irrelevant for steady-state trajectories, i.e., in the limit $\tau_{\mathrm{i}} \rightarrow-\infty$. As $\tau_{\mathrm{i}} \rightarrow-\infty$, the second line vanishes in Eq. 25, because $\kappa_{+} \mathrm{e}^{-\lambda\left(t-\tau_{\mathrm{i}}\right)}-\kappa_{-} \mathrm{e}^{-\lambda\left(2 \tau_{\mathrm{f}}-t-\tau_{\mathrm{i}}\right)} / \kappa_{+}-\kappa_{-} \mathrm{e}^{-2 \lambda\left(\tau_{\mathrm{f}}-\tau_{\mathrm{i}}\right)} \rightarrow 0$. The third line enters into the integral over the initial configuration $\boldsymbol{\eta}_{\mathrm{i}}$ (see Eq.
27) and thus decouples from the trajectory $\overline{\boldsymbol{x}}$ resulting in an irrelevant prefactor. The only relevant contribution as $\tau_{\mathrm{i}} \rightarrow-\infty$ is therefore the first line in Eq. 25 with the integral kernel $\Gamma_{\left[\tau_{\mathrm{i}}, \tau_{\mathrm{f}}\right]}\left(t, t^{\prime}\right)$ reducing to

$$
\Gamma_{\left(-\infty, \tau_{\mathrm{f}}\right]}\left(t, t^{\prime}\right)=\frac{1}{2 \tau_{\mathrm{a}}^{2} \lambda}\left[\mathrm{e}^{-\lambda\left|t-t^{\prime}\right|}-\frac{\kappa_{-}}{\kappa_{+}} \mathrm{e}^{-\lambda\left(2 \tau_{\mathrm{f}}-t-t^{\prime}\right)}\right],
$$

the same expression as $\Gamma_{\left(-\infty, \tau_{\mathrm{f}}\right]}^{\text {ind }}\left(t, t^{\prime}\right)$ from Eq. 10. This illustrates that the system loses its memory about the initial state as $\tau_{\mathrm{i}} \rightarrow-$ $\infty$.

Another comparison to our previous results from Section 3.1 [18] is obtained by plugging the stationary state distribution $p_{\mathrm{i}}\left(\boldsymbol{\eta}_{\mathrm{i}} \mid \boldsymbol{x}_{\mathrm{i}}\right)=p_{\mathrm{ss}}\left(\boldsymbol{\eta}_{\mathrm{i}}\right)=\sqrt{\tau_{\mathrm{a}} / \pi} \mathrm{e}^{-\tau_{\mathrm{a}} \eta_{\mathrm{i}}^{2}}$ into Eq. 27 and performing the Gaussian integral over $\eta_{\mathrm{i}}$. In that case, we should get back the result Eq. 8, Eq. 9 for independent initial conditions. Indeed, including only the terms from Eq. 25 which involve $\boldsymbol{\eta}_{\mathrm{i}}$, we evaluate the Gaussian integral over $\boldsymbol{\eta}_{\mathrm{i}}$, yielding

$$
\begin{aligned}
& \int \mathrm{d} \boldsymbol{\eta}_{\mathrm{i}} \exp \left\{\int _ { \tau _ { \mathrm { i } } } ^ { \tau _ { \mathrm { f } } } \mathrm { d } t \left[\frac{\sqrt{2 D_{\mathrm{a}}}}{2 D}\left[\dot{\boldsymbol{x}}_{t}-\boldsymbol{v}_{t}\right]^{T \kappa_{+} \mathrm{e}^{-\lambda\left(t-\tau_{\mathrm{i}}\right)}-\kappa_{-} \mathrm{e}^{-\lambda\left(2 \tau_{\mathrm{f}}-t-\tau_{\mathrm{i}}\right)}}\right.\right. \\
& \left.\kappa_{+}-\kappa_{-} \mathrm{e}^{-2 \lambda\left(\tau_{\mathrm{f}}-\tau_{\mathrm{i}}\right)}\right] \boldsymbol{\eta}_{\mathrm{i}} \\
& -\frac{\tau_{\mathrm{a}}}{2}\left[2+\frac{\frac{D_{\mathrm{a}}}{D}\left(1-\mathrm{e}^{-2 \lambda\left(\tau_{\mathrm{f}}-\tau_{\mathrm{i}}\right)}\right)}{\left.\left.\kappa_{+}-\kappa_{-} \mathrm{e}^{-2 \lambda\left(\tau_{\mathrm{f}}-\tau_{\mathrm{i}}\right)}\right] \boldsymbol{\eta}_{\mathrm{i}}^{2}\right\}}\right. \\
& =\exp \left\{-\frac{1}{4 D} \int_{\tau_{\mathrm{i}}}^{\tau_{\mathrm{f}}} \mathrm{d} t \int_{\tau_{\mathrm{i}}}^{\tau_{\mathrm{f}}} \mathrm{d} t^{\prime}\left[\dot{\boldsymbol{x}}_{t}-\boldsymbol{v}_{t}\right]^{T}\left[-\frac{D_{\mathrm{a}}}{D} \Gamma_{\left[\tau_{\mathrm{i}}, \tau_{\mathrm{f}}\right]}^{\mathrm{ini}}\left(t, t^{\prime}\right)\right]\left[\dot{\boldsymbol{x}}_{t^{\prime}}-\boldsymbol{v}_{t^{\prime}}\right]\right\}
\end{aligned}
$$

with

$$
\begin{aligned}
& \Gamma_{\left[\tau_{\mathrm{i}}, \tau_{\mathrm{f}}\right]}^{\mathrm{ini}}\left(t, t^{\prime}\right)=\left(\frac{1}{\tau_{\mathrm{a}}}\right) \\
& \times \frac{\kappa_{+}^{2} \mathrm{e}^{-\lambda\left(t+t^{\prime}-2 \tau_{\mathrm{i}}\right)}+\kappa_{-}^{2} \mathrm{e}^{-\lambda\left(4 \tau_{\mathrm{f}}-t-t^{\prime}-2 \tau_{\mathrm{i}}\right)}-2 \kappa_{+} \kappa_{-} \mathrm{e}^{-2 \lambda\left(\tau_{\mathrm{f}}-\tau_{\mathrm{i}}\right)}\left[\mathrm{e}^{\lambda\left(t-t^{\prime}\right)}+\mathrm{e}^{\lambda\left(t^{\prime}-t\right)}\right]}{\left(\kappa_{+}-\kappa_{-} \mathrm{e}^{-2 \lambda\left(\tau_{\mathrm{f}}-\tau_{\mathrm{i}}\right)}\right)\left(\kappa_{+}^{2}-\kappa_{-}^{2} \mathrm{e}^{-2 \lambda\left(\tau_{\mathrm{f}}-\tau_{\mathrm{i}}\right)}\right)} .
\end{aligned}
$$

A somewhat tedious but straightforward calculation then confirms $\Gamma_{\left[\tau_{\mathrm{i}}, \tau_{\mathrm{f}}\right]}\left(t, t^{\prime}\right)+\Gamma_{\left[\tau_{\mathrm{i}}, \tau_{\mathrm{f}}\right]}^{\text {ini }}\left(t, t^{\prime}\right)=\Gamma_{\left[\tau_{\mathrm{i}}, \tau_{\mathrm{f}}\right]}^{\text {ind }}\left(t, t^{\prime}\right)$, as expected.

\section{IRREVERSIBILITY}

In stochastic thermodynamics [2-6], irreversibility is quantified by comparing the probability $\mathfrak{p}[\overline{\boldsymbol{x}}]=\mathfrak{p}\left[\underline{\boldsymbol{x}} \mid \boldsymbol{x}_{\mathrm{i}}\right] p_{\mathrm{i}}\left(\boldsymbol{x}_{\mathrm{i}}\right)$ of observing a specific trajectory $\overline{\boldsymbol{x}}=\{\boldsymbol{x}(t)\}_{t=\tau_{\mathrm{i}}}^{\tau_{\mathrm{f}}}$ in a given experimental setup with the probability $\tilde{\mathfrak{p}}[\overline{\tilde{x}}]$ of observing the exact same trajectory traced out backwards when providing identical experimental conditions. In other words, $\tilde{\mathfrak{p}}[\overline{\tilde{\boldsymbol{x}}}]$ is the probability of observing the "time-reversed" trajectory

$$
\overline{\tilde{\boldsymbol{x}}}=\{\tilde{\boldsymbol{x}}(t)\}_{t=\tau_{\mathrm{i}}}^{\tau_{\mathrm{f}}}=\left\{\boldsymbol{x}\left(\tau_{\mathrm{f}}+\tau_{\mathrm{i}}-t\right)\right\}_{t=\tau_{\mathrm{i}}}^{\tau_{\mathrm{f}}},
$$

with $\tilde{\boldsymbol{x}}\left(\tau_{\mathrm{i}}\right)=\boldsymbol{x}\left(\tau_{\mathrm{f}}\right)$ and $\tilde{\boldsymbol{x}}\left(\tau_{\mathrm{f}}\right)=\boldsymbol{x}\left(\tau_{\mathrm{i}}\right)$, under the time-reversed experimental protocol $\tilde{\boldsymbol{f}}(\boldsymbol{x}, t):=\boldsymbol{f}\left(\boldsymbol{x}, \tau_{\mathrm{f}}+\tau_{\mathrm{i}}-t\right)$ (note that we disregard for convenience the possibility that parts of the forces could be odd under time reversal; it is straightforward to adapt the expressions below accordingly if necessary). For passive Brownian motion, it has been shown that the log-ratio of 
these path probabilities is related to the dissipation occurring along the trajectory $\bar{x}$, quantified as the total change of entropy in the thermal bath and the system. This fundamental connection makes the "irreversibility measure"

$$
\Delta \Sigma[\overline{\boldsymbol{x}}]=-k_{\mathrm{B}} \ln \frac{\tilde{\mathfrak{p}}[\overline{\tilde{\boldsymbol{x}}}]}{\mathfrak{p}[\overline{\boldsymbol{x}}]}
$$

a central quantity of interest also for active particles. Indeed, its connection with dissipation and entropy is under lively debate [16, 18-24].

We here provide a general expression for $\Delta \Sigma$ based on our result Eqs. 25-27 for the path weight $\mathfrak{p}[\overline{\boldsymbol{x}}]$. Since the time-reversed trajectory $\overline{\tilde{\boldsymbol{x}}}$ is supposed to occur under identical conditions as the forward trajectory $\bar{x}$, we can express its probability density via Eqs. 25-27 as well, if we replace $\boldsymbol{v}(\boldsymbol{x}, t)$ by the time-reversed protocol $\tilde{\boldsymbol{v}}(\boldsymbol{x}, t)=\tilde{\boldsymbol{f}}(\boldsymbol{x}, t) / \gamma$ (see below Eq. 31). Using Eq. 31 we then rewrite the path weight for the reversed path in terms of the forward path (and the original protocol $\boldsymbol{v}_{t}=\boldsymbol{v}(\boldsymbol{x}(t), t)$ ). The resulting expression for $\tilde{\mathfrak{p}}[\overline{\tilde{\boldsymbol{x}}}]$ is formally similar to Eq. 25, just with the sign inverted for all $\dot{\boldsymbol{x}}(t)$ terms and all initial coordinates replaced by final ones. Plugging the path weights $\mathfrak{p}[\overline{\boldsymbol{x}}]$ and $\tilde{\mathfrak{p}}[\overline{\tilde{\boldsymbol{x}}}]$ into Eq. 32, and denoting the conditional average over the initial configuration $\boldsymbol{\eta}_{\mathrm{i}}$ of the active fluctuations $\int \mathrm{d} \boldsymbol{\eta}_{\mathrm{i}}(\cdot) p_{\mathrm{i}}\left(\boldsymbol{\eta}_{\mathrm{i}} \mid \boldsymbol{x}_{\mathrm{i}}\right)$ in Eq. 27 by $\langle\cdot\rangle_{\eta_{\mathrm{i}} \mid x_{\mathrm{i}}}$ and the corresponding one over final configurations $\int \mathrm{d} \boldsymbol{\eta}_{\mathrm{f}}(\cdot) p_{\mathrm{f}}\left(\boldsymbol{\eta}_{\mathrm{f}} \mid \boldsymbol{x}_{\mathrm{f}}\right)$ by $\langle\cdot\rangle_{\boldsymbol{\eta}_{\mathrm{f}} \mid \boldsymbol{x}_{\mathrm{f}}}$, we find

$$
\begin{aligned}
& \Delta \Sigma[\overline{\boldsymbol{x}}]=\frac{1}{T} \int_{\tau_{\mathrm{i}}}^{\tau_{\mathrm{f}}} \mathrm{d} t \int_{\tau_{\mathrm{i}}}^{\tau_{\mathrm{f}}} \mathrm{d} t^{\prime} \dot{\boldsymbol{x}}_{t}^{T} \boldsymbol{f}_{t^{\prime}}\left[\delta\left(t-t^{\prime}\right)-\frac{D_{\mathrm{a}}}{D} \Gamma_{\left[\tau_{\mathrm{i}}, \tau_{\mathrm{f}}\right]}\left(t, t^{\prime}\right)\right]-k_{\mathrm{B}} \ln \frac{p\left(\boldsymbol{x}_{\mathrm{f}}\right)}{p\left(\boldsymbol{x}_{\mathrm{i}}\right)} \\
& \left.\left.\left.-k_{\mathrm{B}} \ln \frac{\left\langle\exp \left\{-\int_{\tau_{\mathrm{i}}}^{\tau_{\mathrm{f}}} \mathrm{d} t\left[\frac{\sqrt{2 D_{\mathrm{a}}}}{2 D}\left[\dot{\boldsymbol{x}}_{t}+\boldsymbol{v}_{t}\right]^{\mathrm{T}} \frac{\kappa_{+} \mathrm{e}^{-\lambda\left(t-\tau_{\mathrm{i}}\right)-\kappa_{-}} \mathrm{e}^{-\lambda\left(2 \tau_{\mathrm{f}}-t-\tau_{\mathrm{i}}\right)}}{\kappa_{+}-\kappa_{-} \mathrm{e}^{-2 \lambda\left(\tau_{\mathrm{f}}-\tau_{\mathrm{i}}\right)}} \boldsymbol{\eta}_{\mathrm{f}}\right]\right\}\right\rangle_{\boldsymbol{\eta}_{\mathrm{f}} \mid \boldsymbol{x}_{\mathrm{f}}}}{\left\langle\operatorname { e x p } \left\{+\int_{\tau_{\mathrm{i}}}^{\tau_{\mathrm{f}}} \mathrm{d} t\left[\frac{\sqrt{2 D_{\mathrm{a}}}}{2 D}\left[\dot{\boldsymbol{x}}_{t}-\boldsymbol{v}_{t}\right]^{T} \frac{\kappa_{\mathrm{e}} \mathrm{e}^{-\lambda\left(t-\tau_{\mathrm{i}}\right)-\kappa_{-}-\lambda\left(2 \tau_{\mathrm{f}}-t-\tau_{\mathrm{i}}\right)}}{\kappa_{+}-\kappa_{-} \mathrm{e}^{-2 \lambda}\left(\tau_{\mathrm{f}}-\tau_{\mathrm{i}}\right)}\right.\right.\right.} \boldsymbol{\eta}_{\mathrm{i}}\right]\right\}\right\rangle_{\eta_{\mathrm{i}_{\mathrm{i}}} \mid \boldsymbol{x}_{\mathrm{i}}}
\end{aligned}
$$

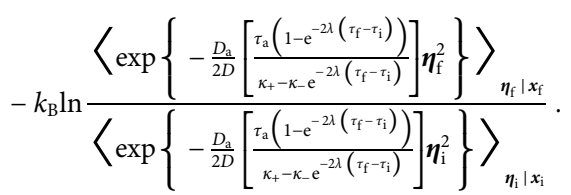

This expression constitutes the second central result of this work. Given any spatial trajectory $\overline{\boldsymbol{x}}=\{\boldsymbol{x}(t)\}_{t=\tau_{\mathrm{j}}}^{\tau_{\mathrm{f}}}$, the measure $\Delta \Sigma[\overline{\boldsymbol{x}}]$ quantifies how irreversible this single trajectory is in the sense of the definition Eq. 32. A trajectory with $\Delta \Sigma[\overline{\boldsymbol{x}}]=0$ is reversible, i.e., movement of the AOUP forward or backward along the trajectory occurs with equal probability, but the larger $\Delta \Sigma[\bar{x}]$ the (exponentially) less likely it is to observe the backward movement.

Central properties of the active fluctuations which drive the particle motion are represented by the parameters $D_{\mathrm{a}}$ (the strength of the active fluctuations) and $\tau_{\mathrm{a}}$ (their correlation time, hidden in $\left.\lambda=\sqrt{1+D_{\mathrm{a}} / D} / \tau_{\mathrm{a}}\right)$. Moreover, our general result Eq. 33 contains averages over the distributions of the active fluctuations $\boldsymbol{\eta}_{\mathrm{i}}$ and $\boldsymbol{\eta}_{\mathrm{f}}$ at the beginning of the particle trajectory and at the beginning of the reversed trajectory (see also Eq. 27). We therefore presuppose that we have some knowledge or control over these distributions when setting up the experiment, even though the (microscopic) degrees of freedom related to the active fluctuations typically are inaccessible, and so are specific realizations of $\boldsymbol{\eta}(t)$ or the specific values of $\boldsymbol{\eta}_{\mathrm{i}}$ and $\boldsymbol{\eta}_{\mathrm{f}}$. For artificial active colloids [10], or in computer experiments, we may imagine, e.g., to let the particles orient randomly before "switching on" the activity, possibly with a specific strength (distribution).

In the spirit of quantifying irreversibility by asking how likely it is to observe a reversed trajectory compared to its forward twin when starting from identically prepared experimental setups (except for the initial particle position, which is $\boldsymbol{x}_{\mathrm{i}}$ for the forward path and $\boldsymbol{x}_{\mathrm{f}}$ for the backward path), we may take the distributions for $\boldsymbol{\eta}_{\mathrm{i}}$ and $\boldsymbol{\eta}_{\mathrm{f}}$ to be the same, or to be "mirror images" of each other under sign-inversion, depending on the physical situation modeled by the active fluctuations $\boldsymbol{\eta}(t)$ (see the discussion in Ref. 18) ${ }^{1}$. Moreover, we may imagine the experiment to be prepared in a way that the initial distributions of the active fluctuations for forward and backward motion are independent of particle positions (a notable exception arising, if the experiment starts from a joint steady state). For such independent initial conditions with identical (or "mirrored") distributions, the third line in Eq. 33 vanishes. The second line, however, is still non-zero, and can be interpreted to quantify the contribution to irreversibility from the initial configuration of the active fluctuations.

The first line in Eq. 33 is independent of $\boldsymbol{\eta}_{\mathrm{i}}$ and $\boldsymbol{\eta}_{\mathrm{f}}$, and thus measures the irreversibility associated with the time-evolution of the spatial particle position alone. It contains three terms (two in the double-integral and a boundary term), which all represent different contributions to irreversibility. The boundary term $-k_{\mathrm{B}} \ln \left[p\left(\boldsymbol{x}_{\mathrm{f}}\right) / p\left(\boldsymbol{x}_{\mathrm{i}}\right)\right]$ does not involve any parameters characterizing the thermal bath or the active fluctuations, and is usually interpreted as the change in system entropy of the AOUP between the beginning and end of the trajectory $\bar{x}$ [18]. The integral involving $\delta\left(t-t^{\prime}\right)$ is independent of the active parameters $D_{\mathrm{a}}$ and $\tau_{\mathrm{a}}$, and is formally identical to the entropy produced in the thermal bath along the trajectory $\bar{x}$ as known for passive Brownian motion [4]. However, in the present case of an AOUP it does not capture the full heat dissipation, because in addition to the force $\boldsymbol{f}(\boldsymbol{x}(t), t)$ also active self-propulsion "forces" $\sqrt{2 D_{\mathrm{a}}} \boldsymbol{\eta}(t)$ drive the particle and contribute to dissipation, i.e., even though the $\delta\left(t-t^{\prime}\right)$-integral can be interpreted as the "thermal contribution" to irreversibility due to the AOUP being in contact with a heat bath, it cannot be identified with the entropy produced in this thermal environment (see also the detailed discussion in Ref. 18). The second, proper doubleintegral encodes the (statistical) characteristics of the active fluctuations via its kernel $\Gamma_{\left[\tau_{i}, \tau_{\mathrm{f}}\right]}\left(t, t^{\prime}\right)$ and, furthermore, vanishes if active propulsion is "switched off", i.e., for $D_{\mathrm{a}}=0$. Hence, it can be interpreted to measure the irreversibility "produced" by the active fluctuations along the trajectory $\overline{\boldsymbol{x}}$, and we will refer to it as the "active contribution" to irreversibility.

${ }^{1}$ In fact, to us these seem to be the only proper choices, if we want $\Delta \Sigma$ to quantify irreversibility. For arbitrary, unrelated distributions of $\boldsymbol{\eta}_{\mathrm{i}}$ and $\boldsymbol{\eta}_{\mathrm{f}}$, we would compare forward and backward paths generated under different experimental conditions. 
These two contributions to irreversibility from the particle trajectory $\overline{\boldsymbol{x}}$, the thermal one and the active one, are non-zero only if external forces $\boldsymbol{f}(\boldsymbol{x}, t)=\gamma \boldsymbol{v}(\boldsymbol{x}, t)$ are present in addition to the active self-propulsion (likewise for the integral in the second line, i.e., for the contribution associated with the initial preparation of the system), implying that the trajectories of "free active motion" appear reversible. For non-conservative forces, both contributions typically lead to a time-extensive increase of irreversibility with the trajectory length $\tau$. For conservative forces $\boldsymbol{f}(\boldsymbol{x})=-\nabla U(\boldsymbol{x})$ derived from a stationary confining potential $U(\boldsymbol{x})$, the thermal contribution reduces to the boundary term $\left[U\left(\boldsymbol{x}_{\mathrm{i}}\right)-U\left(\boldsymbol{x}_{\mathrm{f}}\right)\right] / T$ and thus is non-extensive in $\tau$. Due to the double-integral nature of the active part with the nonlocal kernel $\Gamma_{\left[\tau_{\mathrm{i}}, \tau_{\mathrm{f}}\right]}\left(t, t^{\prime}\right)$ a similarly obvious argument does not apply. Indeed, the question whether or not, or in how far, the trajectory of an AOUP in a confining potential appears (ir)reversible is still not fully answered $[16,18,49]$. We can, however, draw some conclusions from considering the limiting cases of small and large correlations times $\tau_{\mathrm{a}} \rightarrow 0$ and $\tau_{\mathrm{a}} \rightarrow \infty$. In the first case, $\tau_{\mathrm{a}} \rightarrow 0$, the active fluctuations become white and thus behave like a thermal bath, such that the AOUP can be imagined to be a passive Brownian particle in contact with a heat bath at effective temperature $\gamma\left(D+D_{\mathrm{a}}\right) / k_{\mathrm{B}}$, trapped in a confining potential. Accordingly, irreversibility production is non-extensive. In the second case, $\tau_{\mathrm{a}} \rightarrow \infty$, the active fluctuations become constant and thus behave like a bias force which slightly tilts the confining potential. Again, the situation is similar to a trapped passive Brownian particle with non-extensive irreversibility production. We can therefore expect that the active contribution to irreversibility in a confining potential may become maximal at some intermediate value of $\tau_{\mathrm{a}}$.

Another important implication of the result Eq. 33 is that the rate at which irreversibility is produced in the stationary state (i.e., upon letting $\tau_{\mathrm{i}} \rightarrow-\infty$, cf., Section 3.2) is independent of the specific initial distribution $p_{\mathrm{i}}\left(\boldsymbol{x}_{\mathrm{i}}, \boldsymbol{\eta}_{\mathrm{i}}\right)$. Indeed, the terms in the second and third lines of Eq. 33 vanish as $\tau_{\mathrm{i}} \rightarrow-\infty$, and the memory kernel $\Gamma_{\left[\tau_{\mathrm{i}}, \tau_{\mathrm{f}}\right]}\left(t, t^{\prime}\right)$ in the first line assumes the form Eq. 28, independent of the initial distribution (see the discussion in Section 4.2). Hence, if we are only interested in long-time properties, the initial configuration in particular of the self-propulsion drive is irrelevant. While we might have intuitively expected that the long-time irreversibility production rate is independent of the details of the initial setup, it is not completely obvious in the presence of memory effects $[50,51]$. The fact that we can verify it for AOUPs is reassuring though, in so far as control over the initial state is limited in typical active particle systems (as already mentioned above). For infinitely long trajectories $\tau_{\mathrm{f}} \rightarrow \infty$ in the stationary state $\tau_{\mathrm{i}} \rightarrow-\infty$, the expression for $\Delta \Sigma[\overline{\boldsymbol{x}}]$ reduces further to

$$
\Delta \Sigma_{(-\infty, \infty)}[\overline{\boldsymbol{x}}]=\frac{1}{T} \int_{\tau_{\mathrm{i}}}^{\tau_{\mathrm{f}}} \mathrm{d} t \int_{\tau_{\mathrm{i}}}^{\tau_{\mathrm{f}}} \mathrm{d} t^{\prime} \dot{\boldsymbol{x}}_{t}^{T} \boldsymbol{f}_{t^{\prime}}\left[\delta\left(t-t^{\prime}\right)-\frac{D_{\mathrm{a}}}{D} \Gamma_{(-\infty, \infty)}\left(t, t^{\prime}\right)\right]
$$

with $\Gamma_{(-\infty, \infty)}\left(t, t^{\prime}\right)$ from Eq. 11 (see the discussion around Eq. 28), in agreement with the findings in Refs. 24 and 48.
We conclude this discussion with a remark concerning the relation between the expression for $\Delta \Sigma[\overline{\boldsymbol{x}}]$ found earlier in Ref. 18 and the result Eq. 33 derived here. In Ref. 18, we have calculated $\Delta \Sigma[\overline{\boldsymbol{x}}]$ under the assumption that the active fluctuations are in their stationary state, independent of initial particle positions, at the beginning of the forward path and at the beginning of the backward path, i.e., $p_{\mathrm{i}}\left(\boldsymbol{\eta}_{\mathrm{i}} \mid \boldsymbol{x}_{\mathrm{i}}\right)=p_{\mathrm{ss}}\left(\boldsymbol{\eta}_{\mathrm{i}}\right)=\sqrt{\tau_{\mathrm{a}} / \pi} \mathrm{e}^{-\tau_{\mathrm{a}} \eta_{\mathrm{i}}^{2}}$ and $p_{\mathrm{f}}\left(\boldsymbol{\eta}_{\mathrm{f}} \mid \boldsymbol{x}_{\mathrm{f}}\right)=p_{\mathrm{ss}}\left(\boldsymbol{\eta}_{\mathrm{f}}\right)=\sqrt{\tau_{\mathrm{a}} / \pi} \mathrm{e}^{-\tau_{\mathrm{a}}} \boldsymbol{\eta}_{\mathrm{f}}^{2}$. The resulting expression for Eq. 33 looks formally identical to the first line in Eq. 33, but with $\Gamma_{\left[\tau_{\mathrm{i}}, \tau_{\mathrm{f}}\right]}\left(t, t^{\prime}\right)$ substituted by $\Gamma_{\left[\tau_{\mathrm{i}}, \tau_{\mathrm{f}}\right]}^{\text {ind }}\left(t, t^{\prime}\right)$ from Eq. 8 (compare with eqs. $42 \mathrm{~b}$ and $40 \mathrm{a}$ in Ref. 18). As we can see from the calculation at the end of Section 4.2 above, the "amount of irreversibility" stemming from the particular stationary-state initial configuration of the active fluctuations has been absorbed into $\Gamma_{\left[\tau_{\mathrm{i}}, \tau_{\mathrm{f}}\right]}^{\text {ind }}\left(t, t^{\prime}\right)$, and is therefore not explicitly visible in Ref. 18 as an additional term analogous to the second line in Eq. 33.

\section{CONCLUSION}

What can we learn about the non-equilibrium nature of an active system by observing particle trajectories, i.e., the evolution of particle positions over time? Within the framework of a minimal model for particulate active matter on the micro- and nanoscale, the active Ornstein-Uhlenbeck particle [15, 25-31] (see Eqs. 1 and 2), we here contribute an essential step toward exploring this question by deriving an exact analytical expression for the path weight (Eqs. 25-27), which is valid for any values of the model parameters, any external driving forces, arbitrary initial particle positions and configurations of the active fluctuations, and arbitrary trajectory durations. We use this general expression to calculate the logratio of path weights for forward vs. backward trajectories (see Eq. 33). In analogy to the stochastic thermodynamics of passive Brownian particles [2-6], such an irreversibility measure may provide an approach toward a thermodynamic description of active matter [16, 18-24].

In future works we may build on these results to further explore the non-equilibrium properties of AOUPs. A highly interesting problem is a possible thermodynamic interpretation of the path probability ratio $\Delta \Sigma[\overline{\boldsymbol{x}}][18]$, e.g., via exploring its connection to active pressure $[14,52]$, to the different phases observed in active matter [53], or to the arrow of time [54,55] in these systems. Such a thermodynamic interpretation, in particular concerning the role of dissipation, may finally allow to quantify efficiency fluctuations in stochastic heat engines operating between active baths [56], in analogy to passive stochastic heat engines [57-59]. Other important questions which can be approached directly by using our general result for the path weight $\mathfrak{p}[\overline{\boldsymbol{x}}]$ include the analysis of the response behavior under external perturbations [43] or of violations of the fluctuation-response relation $[60,61]$ due to the inherent nonequilibrium character of active matter, and their potential for probing properties of the active fluctuations [61]. Finally, it would be interesting to explore if our analytical methods used here to integrate out the simple Ornstein-Uhlenbeck fluctuations Eq. 2 
can be extended to treat more general active fluctuations, like the ones considered in Ref. 62.

\section{DATA AVAILABILITY STATEMENT}

The original contributions presented in the study are included in the article/Supplementary Material, further inquiries can be directed to the corresponding authors.

\section{AUTHOR CONTRIBUTIONS}

All authors contributed equally to this work.

\section{REFERENCES}

1. Callen HB. Thermodynamics \& an introduction to thermostatistics. Hoboken, NJ: John Wiley \& Sons (2006). $512 \mathrm{p}$.

2. Seifert U. Stochastic thermodynamics: principles and perspectives. Eur Phys J B. (2008). 64:423-31. doi:10.1140/epjb/e2008-00001-9

3. Jarzynski C. Equalities and inequalities: irreversibility and the second law of thermodynamics at the nanoscale. Annu Rev Condens Matter Phys. (2011). 2: 329-51. doi:10.1146/annurev-conmatphys-062910-140506

4. Seifert U. Stochastic thermodynamics, fluctuation theorems and molecular machines. Rep Prog Phys. (2012). 75:126001. doi:10.1088/0034-4885/75/12/ 126001

5. Van den Broeck C, Esposito M. Ensemble and trajectory thermodynamics: a brief introduction. Phys Stat Mech Appl. (2015). 418:6-16. doi:10.1016/j.physa. 2014.04.035

6. Seifert U. Stochastic thermodynamics: from principles to the cost of precision. Phys Stat Mech Appl. (2018). 504:176-91. doi:10.1016/j.physa.2017.10.024

7. Romanczuk P, Bär M, Ebeling W, Lindner B, Schimansky-Geier L. Active brownian particles. Eur Phys J Spec Top. (2012). 202:1-162. doi:10.1140/epjst/ e2012-01529-y

8. Cates ME. Diffusive transport without detailed balance in motile bacteria: does microbiology need statistical physics? Rep Prog Phys. (2012). 75:042601. doi:10. 1088/0034-4885/75/4/042601

9. Elgeti J, Winkler RG, Gompper G. Physics of microswimmers-single particle motion and collective behavior: a review. Rep Prog Phys. (2015). 78:056601. doi:10.1088/0034-4885/78/5/056601

10. Bechinger C, Di Leonardo R, Löwen H, Reichhardt C, Volpe G, Volpe G. Active particles in complex and crowded environments. Rev Mod Phys. (2016). 88:045006. doi:10.1103/RevModPhys.88.045006

11. Patteson AE, Gopinath A, Arratia PE. Active colloids in complex fluids. Curr Opin Colloid Interface Sci. (2016). 21:86-96. doi:10.1016/j.cocis.2016.01.001

12. Tailleur J, Cates ME. Statistical mechanics of interacting run-and-tumble bacteria. Phys Rev Lett. (2008). 100:218103. doi:10.1103/PhysRevLett.100. 218103

13. Speck T, Bialké J, Menzel AM, Löwen H. Effective cahn-hilliard equation for the phase separation of active brownian particles. Phys Rev Lett. (2014). 112: 218304. doi:10.1103/physrevlett.112.218304

14. Takatori SC, Yan W, Brady JF. Swim pressure: stress generation in active matter. Phys Rev Lett. (2014). 113:028103. doi:10.1103/physrevlett.113.028103

15. Farage TFF, Krinninger P, Brader JM. Effective interactions in active brownian suspensions. Phys Rev E. (2015). 91:042310. doi:10.1103/PhysRevE.91.042310

16. Fodor É, Nardini C, Cates ME, Tailleur J, Visco P, van Wijland F. How far from equilibrium is active matter? Phys Rev Lett. (2016). 117:038103. doi:10.1103/ PhysRevLett.117.038103

17. Nardini C, Fodor É, Tjhung E, van Wijland F, Tailleur J, Cates ME. Entropy production in field theories without time-reversal symmetry: quantifying the non-equilibrium character of active matter. Phys Rev X. (2017). 7:021007. doi:10.1103/physrevx.7.021007

\section{FUNDING}

This research has been funded by the Swedish Research Council (Vetenskapsrådet) under the Grants No. 201605412 (RE) and by the Deutsche Forschungsgemeinschaft (DFG) within the Research Unit FOR 2692 under Grant No. 397303734 (LD).

\section{ACKNOWLEDGMENTS}

We thank Stefano Bo for stimulating discussion. LD gratefully acknowledges support by the Nordita visiting PhD program.

18. Dabelow L, Bo S, Eichhorn R. Irreversibility in active matter systems: fluctuation theorem and mutual information. Phys Rev X. (2019). 9:021009. doi:10.1103/physrevx.9.021009

19. Marconi UMB, Puglisi A, Maggi C. Heat, temperature and clausius inequality in a model for active brownian particles. Sci Rep. (2017). 7:46496. doi:10.1038/ srep46496

20. Mandal D, Klymko K, DeWeese MR. Entropy production and fluctuation theorems for active matter. Phys Rev Lett. (2017). 119:258001. doi:10.1103/ physrevlett.119.258001

21. Puglisi A, Marconi UMB. Clausius relation for active particles: what can we learn from fluctuations. Entropy. (2017). 19:356. doi:10.3390/e19070356

22. Caprini L, Marconi UMB, Puglisi A, Vulpiani A. Comment on "entropy production and fluctuation theorems for active matter". Phys Rev Lett. (2018). 121:139801. doi:10.1103/physrevlett.121.139801

23. Mandal D, Klymko K, DeWeese MR. A reply to the comment by Mandal, Klymko, and DeWeese. Phys Rev Lett. (2018). 121:139802. doi:10.1103/ physrevlett.121.139802

24. Caprini L, Marconi UMB, Puglisi A, Vulpiani A. The entropy production of Ornstein-Uhlenbeck active particles: a path integral method for correlations. J Stat Mech. (2019). 2019:053203. doi:10.1088/1742-5468/ab14dd

25. Fily Y, Marchetti MC. Athermal phase separation of self-propelled particles with no alignment. Phys Rev Lett. (2012). 108:235702. doi:10.1103/ PhysRevLett.108.235702

26. Maggi C, Paoluzzi M, Pellicciotta N, Lepore A, Angelani L, Di Leonardo R. Generalized energy equipartition in harmonic oscillators driven by active baths. Phys Rev Lett. (2014). 113:238303. doi:10.1103/PhysRevLett.113. 238303

27. Argun A, Moradi A-R, Pinçe E, Bagci GB, Imparato A, Volpe G. NonBoltzmann stationary distributions and nonequilibrium relations in active baths. Phys Rev E. (2016). 94:062150. doi:10.1103/PhysRevE.94.062150

28. Maggi C, Paoluzzi M, Angelani L, Di Leonardo R. Memory-less response and violation of the fluctuation-dissipation theorem in colloids suspended in an active bath. Sci Rep. (2017). 7:17588. doi:10.1038/s41598-017-17900-2

29. Chaki S, Chakrabarti R. Entropy production and work fluctuation relations for a single particle in active bath. Phys Stat Mech Appl. (2018). 511:302-15. doi:10. 1016/j.physa.2018.07.055

30. Marconi UMB, Maggi C. Towards a statistical mechanical theory of active fluids. Soft Matter. (2015). 11:8768-81. doi:10.1039/C5SM01718A

31. Shankar S, Marchetti MC. Hidden entropy production and work fluctuations in an ideal active gas. Phys Rev E. (2018). 98:020604. doi:10.1103/physreve.98. 020604

32. Koumakis N, Maggi C, Di Leonardo R. Directed transport of active particles over asymmetric energy barriers. Soft Matter. (2014). 10:5695-701. doi:10. $1039 / \mathrm{c} 4 \mathrm{sm} 00665 \mathrm{~h}$

33. Szamel G. Self-propelled particle in an external potential: existence of an effective temperature. Phys Rev E. (2014). 90:012111. doi:10.1103/physreve.90.012111

34. Szamel G, Flenner E, Berthier L. Glassy dynamics of athermal self-propelled particles: computer simulations and a nonequilibrium microscopic theory. Phys Rev E. (2015). 91:062304. doi:10.1103/physreve.91.062304 
35. Maggi C, Marconi UMB, Gnan N, Di Leonardo R. Multidimensional stationary probability distribution for interacting active particles. Sci Rep. (2015). 5:10742. doi:10.1038/srep10742

36. Flenner E, Szamel G, Berthier L. The nonequilibrium glassy dynamics of selfpropelled particles. Soft Matter. (2016). 12:7136-49. doi:10.1039/ C6SM01322H

37. Paoluzzi M, Maggi C, Marconi UMB, Gnan N. Critical phenomena in active matter. Phys Rev E. (2016). 94:052602. doi:10.1103/physreve.94.052602

38. Marconi UMB, Gnan N, Paoluzzi M, Maggi C, Di Leonardo R. Velocity distribution in active particles systems. Sci Rep. (2016). 6:23297. doi:10.1038/srep26215

39. Szamel G. Evaluating linear response in active systems with no perturbing field. Europhys Lett. (2017). 117:50010. doi:10.1209/0295-5075/117/50010

40. Sandford C, Grosberg AY, Joanny JF. Pressure and flow of exponentially selfcorrelated active particles. Phys Rev E. (2017). 96:052605. doi:10.1103/ physreve.96.052605

41. Caprini L, Marconi UMB, Vulpiani A. Linear response and correlation of a self-propelled particle in the presence of external fields. J Stat Mech. (2018). 2018:033203. doi:10.1088/1742-5468/aaa78c

42. Fodor É, Cristina Marchetti M. The statistical physics of active matter: from self-catalytic colloids to living cells. Phys Stat Mech Appl. (2018). 504:106-20. doi:10.1016/j.physa.2017.12.137

43. Dal Cengio S, Levis D, Pagonabarraga I. Linear response theory and greenkubo relations for active matter. Phys Rev Lett. (2019). 123:238003. doi:10. 1103/physrevlett.123.238003

44. Bonilla L. Active ornstein-uhlenbeck particles. Phys Rev. (2019). 100:022601. doi:10.1103/physreve.100.022601

45. Onsager L, Machlup S. Fluctuations and irreversible processes. Phys Rev. (1953). 91:1505-12. doi:10.1103/PhysRev.91.1505

46. Machlup S, Onsager L. Fluctuations and irreversible process. II. Systems with kinetic energy. Phys Rev. (1953). 91:1512-5. doi:10.1103/PhysRev.91.1512

47. Cugliandolo LF, Lecomte V. Rules of calculus in the path integral representation of white noise Langevin equations: the Onsager-Machlup approach. J Phys Math Theor. (2017). 50:345001. doi:10.1088/1751-8121/aa7dd6

48. Zamponi F, Bonetto F, Cugliandolo LF, Kurchan J. A fluctuation theorem for non-equilibrium relaxational systems driven by external forces. J. Stat. Mech. (2005). 2005:P09013. doi:10.1088/1742-5468/2005/09/p09013

49. Dabelow L, Bo S, Eichhorn R. How irreversible are steady-state trajectories of a trapped active particle? (unpublished).

50. Harris RJ, Touchette H. Current fluctuations in stochastic systems with longrange memory. J Phys Math Theor. (2009). 42:342001. doi:10.1088/1751-8113/ $42 / 34 / 342001$

51. Puglisi A, Villamaina D. Irreversible effects of memory. Europhys Lett. (2009). 88:30004. doi:10.1209/0295-5075/88/30004
52. Solon AP, Stenhammar J, Wittkowski R, Kardar M, Kafri Y, Cates ME, et al. Pressure and phase equilibria in interacting active brownian spheres. Phys Rev Lett. (2015). 114:198301. doi:10.1103/physrevlett.114.198301

53. Cates ME, Tailleur J. Motility-induced phase separation. Annu Rev Condens Matter Phys. (2015). 6:219-44. doi:10.1146/annurev-conmatphys-031214014710

54. Roldán É, Neri I, Dörpinghaus M, Meyr H, Jülicher F. Decision making in the arrow of time. Phys Rev Lett. (2015). 115:250602. doi:10.1103/physrevlett.115. 250602

55. Roldán É, Barral J, Martin P, Parrondo JM, Jülicher F. Arrow of time in active fluctuations. Preprint repository name [Preprint] (2018). Available from: https://arxiv.org/abs/1803.04743.

56. Krishnamurthy S, Ghosh S, Chatterji D, Ganapathy R, Sood AK. A micrometre-sized heat engine operating between bacterial reservoirs. Nat Phys. (2016). 12:1134-8. doi:10.1038/nphys3870

57. Verley G, Esposito M, Willaert T, Van den Broeck C. The unlikely Carnot efficiency. Nat Commun. (2014). 5:4721. doi:10.1038/ncomms5721

58. Verley G, Willaert T, Van den Broeck C, Esposito M. Universal theory of efficiency fluctuations. Phys Rev E. (2014). 90:052145. doi:10.1103/physreve. 90.052145

59. Manikandan SK, Dabelow L, Eichhorn R, Krishnamurthy S. Efficiency fluctuations in microscopic machines. Phys Rev Lett. (2019). 122:140601. doi:10.1103/physrevlett.122.140601

60. Harada T, Si S. Equality connecting energy dissipation with a violation of the fluctuation-response relation. Phys Rev Lett. (2005). 95:130602. doi:10.1103/ physrevlett.95.130602

61. Gnesotto FS, Mura F, Gladrow J, Broedersz CP. Broken detailed balance and non-equilibrium dynamics in living systems: a review. Rep Prog Phys. (2018). 81:066601. doi:10.1088/1361-6633/aab3ed

62. Sevilla FJ, Rodríguez RF, Gomez-Solano JR. Generalized Ornstein-Uhlenbeck model for active motion. Phys Rev E. (2019). 100:032123. doi:10.1103/ physreve.100.032123

Conflict of Interest: The authors declare that the research was conducted in the absence of any commercial or financial relationships that could be construed as a potential conflict of interest.

Copyright (c) 2021 Eichhorn and Dabelow. This is an open-access article distributed under the terms of the Creative Commons Attribution License (CC BY). The use, distribution or reproduction in other forums is permitted, provided the original author(s) and the copyright owner(s) are credited and that the original publication in this journal is cited, in accordance with accepted academic practice. No use, distribution or reproduction is permitted which does not comply with these terms. 


\section{APPENDIX}

\section{Evaluation of $\Gamma_{\sigma}\left(\mathbf{t}, \mathbf{t}^{\prime}\right)$}

We here outline the calculation of $\Gamma_{\sigma}\left(t, t^{\prime}\right)$ as the inverse of the differential operator

$$
\begin{aligned}
& V_{\sigma}\left(t, t^{\prime}\right):=\delta\left(t-t^{\prime}\right)\left[-\tau_{\mathrm{a}}^{2} \partial_{t^{\prime}}^{2}+1+\frac{D_{\mathrm{a}}}{D}+\delta\left(t^{\prime}\right)\right. \\
& \left.\left(-\tau_{\mathrm{a}}^{2} \partial_{t^{\prime}}-\tau_{\mathrm{a}}+\frac{1}{\sigma^{2}}\right)+\delta\left(\tau-t^{\prime}\right)\left(\tau_{\mathrm{a}}^{2} \partial_{t^{\prime}}+\tau_{\mathrm{a}}\right)\right],
\end{aligned}
$$

i.e., $\Gamma_{\sigma}\left(t, t^{\prime}\right)$ is a solution of the equation $\int_{0}^{\tau} \mathrm{d} t^{\prime} V_{\sigma}\left(t, t^{\prime}\right) \Gamma_{\sigma}\left(t^{\prime}, t^{\prime \prime}\right)=\delta\left(t-t^{\prime \prime}\right)$. In fact, the operator $V_{\sigma}\left(t, t^{\prime}\right)$ is "diagonal" in the time arguments such that $\Gamma_{\sigma}\left(t, t^{\prime}\right)$ solves the differential equation

$$
\begin{aligned}
& {\left[-\tau_{\mathrm{a}}^{2} \partial_{t}^{2}+1+\frac{D_{\mathrm{a}}}{D}+\delta(t)\left(-\tau_{\mathrm{a}}^{2} \partial_{t}-\tau_{\mathrm{a}}+\frac{1}{\sigma^{2}}\right)\right.} \\
& \left.+\delta(\tau-t)\left(\tau_{\mathrm{a}}^{2} \partial_{t}+\tau_{\mathrm{a}}\right)\right] \Gamma_{\sigma}\left(t, t^{\prime}\right)=\delta\left(t-t^{\prime}\right) .
\end{aligned}
$$

Note that $t^{\prime}$ is essentially a fixed parameter here, just like $D, D_{\mathrm{a}}, \tau_{\mathrm{a}}$ and $\sigma$. To find the solution, we follow the procedure from Ref. 18, i.e., we compose $\Gamma_{\sigma}\left(t, t^{\prime}\right)$ from two parts as $\Gamma_{\sigma}\left(t, t^{\prime}\right)=G\left(t, t^{\prime}\right)+H_{\sigma}\left(t, t^{\prime}\right)$. First, we construct the function $G\left(t, t^{\prime}\right)$ as the Green's function solving the equation $\left[-\tau_{a}^{2} \partial_{t}^{2}+\left(1+D_{\mathrm{a}} / D\right)\right] G\left(t, t^{\prime}\right)=\delta\left(t-t^{\prime}\right)$ with homogeneous boundary conditions $G\left(0, t^{\prime}\right)=G\left(\tau, t^{\prime}\right)=0$. Second, we determine $H_{\sigma}\left(t, t^{\prime}\right)$ as a solution of the homogeneous problem $\left[-\tau_{a}^{2} \partial_{t}^{2}+\left(1+D_{\mathrm{a}} / D\right)\right] H_{\sigma}\left(t, t^{\prime}\right)=0$ such that the boundary terms are fixed as prescribed by Eq. 36.

We can construct both parts, $G\left(t, t^{\prime}\right)$ and $H_{\sigma}\left(t, t^{\prime}\right)$, from the general solution

$$
\Gamma(t)=a^{+} \mathrm{e}^{\lambda t}+a^{-} \mathrm{e}^{-\lambda t}, \quad \lambda=\frac{1}{\tau_{\mathrm{a}}} \sqrt{1+\frac{D_{\mathrm{a}}}{D}}, \quad a^{ \pm}=\mathrm{const}
$$

of the homogeneous ordinary differential equation

$$
\left[-\tau_{\mathrm{a}}^{2} \partial_{t}^{2}+1+\frac{D_{\mathrm{a}}}{D}\right] \Gamma(t)=0
$$

associated with Eq. 36. The Green's function $G\left(t, t^{\prime}\right)$ is exactly the same as in Ref. 18. Accordingly, its construction is completely analogous to the procedure outlined in Appendix B of Ref. 18, and we only recall the result here,

$$
G\left(t, t^{\prime}\right)=\frac{1}{2 \tau_{\mathrm{a}}^{2} \lambda} \frac{\mathrm{e}^{\lambda\left(\tau-\left|t-t^{\prime}\right|\right)}-\mathrm{e}^{\lambda\left(\tau-t-t^{\prime}\right)}+\mathrm{e}^{-\lambda\left(\tau-\left|t-t^{\prime}\right|\right)}-\mathrm{e}^{-\lambda\left(\tau-t-t^{\prime}\right)}}{\mathrm{e}^{\lambda \tau}-\mathrm{e}^{-\lambda \tau}} .
$$

The difference between the present calculation and the one in Ref. 18 is the boundary term at $t=0$, which contains a contribution from the arbitrary (Gaussian) initial distribution of the active fluctuations. To take both boundary terms in Eq. 36 into account, we make an ansatz of the form Eq. 37 for the function $H_{\sigma}\left(t, t^{\prime}\right)$, i.e., $H_{\sigma}\left(t, t^{\prime}\right)=a^{+} \mathrm{e}^{\lambda t}+a^{-} \mathrm{e}^{-\lambda t}$. The coefficients $a^{ \pm}$are fixed by ensuring that the full solution $\Gamma_{\sigma}\left(t, t^{\prime}\right)=G\left(t, t^{\prime}\right)+$ $H_{\sigma}\left(t, t^{\prime}\right)$ fulfills Eq. 36. Plugging $G\left(t, t^{\prime}\right)+H_{\sigma}\left(t, t^{\prime}\right)$ into Eq. 36, and using $\left[-\tau_{a}^{2} \partial_{t}^{2}+\left(1+D_{\mathrm{a}} / D\right)\right] G\left(t, t^{\prime}\right)=\delta\left(t-t^{\prime}\right)$ and $\left[-\tau_{\mathrm{a}}^{2} \partial_{t}^{2}+1+\frac{D_{\mathrm{a}}}{D}\right] H_{\sigma}\left(t, t^{\prime}\right)=0$, we are left with

$$
\begin{aligned}
& \delta(t)\left[-\left.\tau_{\mathrm{a}}^{2} \partial_{t} G\left(t, t^{\prime}\right)\right|_{t=0}+a^{+}\left(1 / \sigma^{2}-\tau_{\mathrm{a}} \kappa_{+}\right)+a^{-}\left(1 / \sigma^{2}-\tau_{\mathrm{a}} \kappa_{-}\right)\right] \\
& +\delta(\tau-t)\left[\left.\tau_{\mathrm{a}}^{2} \partial_{t} G\left(t, t^{\prime}\right)\right|_{t=\tau}+a^{+} \tau_{\mathrm{a}} \kappa_{+} \mathrm{e}^{\lambda \tau}+a^{-} \tau_{\mathrm{a}} \kappa_{-} \mathrm{e}^{-\lambda \tau}\right]=0,
\end{aligned}
$$

where $\kappa_{ \pm}=1 \pm \lambda \tau_{\mathrm{a}}=1 \pm \sqrt{1+D_{\mathrm{a}} / D}$. Requiring that the terms in the two square brackets each vanish, we can solve for the coefficients $a^{ \pm}$, yielding

$a^{+}=\left(\frac{1}{\tau_{\mathrm{a}}}\right) \frac{\left(1-\sigma^{2} \tau_{\mathrm{a}} \kappa_{-}\right)\left[\mathrm{e}^{-\lambda\left(2 \tau-t^{\prime}\right)}-\mathrm{e}^{-\lambda\left(2 \tau+t^{\prime}\right)}\right]-\kappa_{-}\left[\mathrm{e}^{-\lambda\left(2 \tau+t^{\prime}\right)}-\mathrm{e}^{-\lambda\left(4 \tau-t^{\prime}\right)}\right]}{\kappa_{+}\left(1-\sigma^{2} \tau_{\mathrm{a}} \kappa_{-}\right)-\kappa_{-}\left(1-\sigma^{2} \tau_{\mathrm{a}} \kappa_{+}\right) \mathrm{e}^{-2 \lambda \tau}}$

$$
a^{-}=\left(\frac{1}{\tau_{\mathrm{a}}}\right) \frac{-\left(1-\sigma^{2} \tau_{\mathrm{a}} \kappa_{+}\right)\left[\mathrm{e}^{-\lambda\left(2 \tau-t^{\prime}\right)}-\mathrm{e}^{-\lambda\left(2 \tau+t^{\prime}\right)}\right]+\kappa_{+}\left[\mathrm{e}^{-\lambda t^{\prime}}-\mathrm{e}^{-\lambda\left(2 \tau-t^{\prime}\right)}\right]}{\kappa_{+}\left(1-\sigma^{2} \tau_{\mathrm{a}} \kappa_{-}\right)-\kappa_{-}\left(1-\sigma^{2} \tau_{\mathrm{a}} \kappa_{+}\right) \mathrm{e}^{-2 \lambda \tau}} .
$$

Substituting these coefficients into the above ansatz for $H_{\sigma}\left(t, t^{\prime}\right)$ [see below Eq. 39] and combining it with $G\left(t, t^{\prime}\right)$ from Eq. 39 according to $\Gamma_{\sigma}\left(t, t^{\prime}\right)=G\left(t, t^{\prime}\right)+H_{\sigma}\left(t, t^{\prime}\right)$, we obtain the result stated in Eq. 17 of the main text. 